\title{
Search for the Decay of the Higgs Boson to Charm Quarks with the ATLAS Experiment
}

\author{
M. Aaboud et al." \\ (ATLAS Collaboration)
}

(Received 14 February 2018; published 22 May 2018)

\begin{abstract}
A direct search for the standard model Higgs boson decaying to a pair of charm quarks is presented. Associated production of the Higgs and $\mathrm{Z}$ bosons, in the decay mode $\mathrm{ZH} \rightarrow \ell^{+} \ell^{-} c \bar{c}$ is studied. A data set with an integrated luminosity of $36.1 \mathrm{fb}^{-1}$ of $p p$ collisions at $\sqrt{s}=13 \mathrm{TeV}$ recorded by the ATLAS experiment at the LHC is used. The $H \rightarrow c \bar{c}$ signature is identified using charm-tagging algorithms. The observed (expected) upper limit on $\sigma(p p \rightarrow Z H) \times \mathcal{B}(H \rightarrow c \bar{c})$ is $2.7\left(3.9_{-1.1}^{+2.1}\right)$ pb at the $95 \%$ confidence level for a Higgs boson mass of $125 \mathrm{GeV}$, while the standard model value is $26 \mathrm{fb}$.
\end{abstract}

DOI: $10.1103 /$ PhysRevLett.120.211802

In July 2012, the ATLAS and CMS collaborations announced the discovery of a new particle with a mass of approximately $125 \mathrm{GeV}[1,2]$ in searches for the standard model (SM) Higgs boson at the Large Hadron Collider (LHC) [3]. Subsequent measurements indicate that this particle is consistent with the SM Higgs boson [4-10]. Direct evidence for the Yukawa coupling of the Higgs boson to the top [11] and bottom [12,13] quarks was recently obtained. Measurements of the Yukawa coupling of the Higgs boson to quarks in generations other than the third are difficult at hadron colliders, due to small branching fractions, large backgrounds, and challenges in jet flavor identification $[14,15]$. This Letter presents a direct search by the ATLAS experiment for the decay of the Higgs boson to a pair of charm $(c)$ quarks. This search targets the production of the Higgs boson in association with a $Z$ boson decaying to charged leptons: $Z\left(\ell^{+} \ell^{-}\right) H(c \bar{c})$, where $\ell=e, \mu$.

The SM branching fraction for a Higgs boson with a mass of $125 \mathrm{GeV}$ to decay to a pair of charm quarks is predicted to be $2.9 \%$ [16]. The inclusive cross section for $\sigma(p p \rightarrow Z H) \times \mathcal{B}(H \rightarrow c \bar{c})$ is $26 \mathrm{fb}$ at $\sqrt{s}=13 \mathrm{TeV}$ [17]. Rare exclusive decays of the Higgs boson to a light vector meson or quarkonium state and a photon can also probe the couplings of the second-generation quarks to the Higgs boson [18-21]. Previously, the ATLAS Collaboration presented an indirect search for the decay of the Higgs boson to $c$ quarks via the decay to $J / \psi \gamma$, obtaining a branching fraction limit of $1.5 \times 10^{-3}$ at the $95 \%$ confidence level (C.L.), which approximately corresponds to a limit of 540 times the SM branching fraction prediction

\footnotetext{
*Full author list given at the end of the article.

Published by the American Physical Society under the terms of the Creative Commons Attribution 4.0 International license. Further distribution of this work must maintain attribution to the author(s) and the published article's title, journal citation, and DOI. Funded by SCOAP ${ }^{3}$.
}

[14,20]. Bounds on the Higgs boson branching fractions to unobserved final states and fits to global rates constrain $\mathcal{B}(H \rightarrow c \bar{c})<20 \%$ at the $95 \%$ C.L., assuming SM production cross sections [22]. These limits can still accommodate large modifications to the Higgs boson coupling to charm quarks from new physics [22]. In this Letter, a new approach is introduced to investigate the coupling of the Higgs boson to charm quarks.

The search is performed using $p p$ collision data recorded in 2015 and 2016 with the ATLAS detector [23] at $\sqrt{s}=13 \mathrm{TeV}$. The ATLAS detector at the LHC covers nearly the entire solid angle around the collision point [24]. It consists of an inner tracking detector surrounded by a thin superconducting solenoid, electromagnetic and hadronic calorimeters, and a muon spectrometer incorporating three large superconducting toroidal magnets. An additional pixel layer was installed for the $\sqrt{s}=13 \mathrm{TeV}$ running period [25]. After the application of beam, detector, and data-quality requirements, the integrated luminosity corresponds to $36.1 \pm 0.8 \mathrm{fb}^{-1}$, measured following Ref. [26]. Events are required to contain exactly two sameflavor leptons with an invariant mass consistent with that of the $Z$ boson, and at least two jets of which one or two are identified as charm jets ( $c$ jets). In this Letter, lepton refers to only electrons or muons. The analysis procedure is validated by measuring the yield of $Z W$ and $Z Z$ production, where the sample is enriched in $W \rightarrow c s, c d$ and $Z \rightarrow c \bar{c}$ decays. Further details can be found in Ref. [12].

Monte Carlo (MC) simulated samples were produced for signal and background processes using the full ATLAS detector simulation [27] using GEANT4 [28]. Table I provides details of the event generators used for each signal and background sample. Signal events were produced at next-to-leading order (NLO) for the $q \bar{q} \rightarrow Z H$ process and at leading order (LO) for the $g g \rightarrow Z H$ process with POWHEG-BOX v2 [32]. The dominant $Z+$ jets background and the resonant diboson $Z W$ and $Z Z$ processes were generated using SHERPA 2.2.1 [54]. The $t \bar{t}$ background was 
TABLE I. The configurations used for event generation of the signal and background processes. If two parton distribution functions (PDFs) are shown, the first is for the matrix element calculation and the second for the parton shower, otherwise the same is used for both. Alternative event generators and configurations, used to estimate systematic uncertainties, are in parentheses. Tune refers to the underlying-event tuned parameters of the parton shower event generator. MG5_AMC refers to MADGRAPH5_AMC@NLO 2.2.2 [29]; PYTHIA 8 refers to version 8.212 [30]. Heavy-flavor hadron decays modeled by EvTGEN 1.2.0 [31] are used for all samples except those generated using SHERPA. The order of the calculation of the cross sections used to normalize the predictions is indicated. The $q \bar{q} \rightarrow Z H$ cross section is estimated by subtracting the $g g \rightarrow Z H$ cross section from the $p p \rightarrow Z H$ cross section. The asterisk (*) in the last column denotes that the indicated order is for the $p p \rightarrow Z H$ cross section. NNLO denotes next-to-next-to-leading order; NLL denotes next-toleading log and NNLL denotes next-to-next-to-leading log.

\begin{tabular}{|c|c|c|c|c|c|}
\hline Process & $\begin{array}{l}\text { Event Generator } \\
\text { (alternative) }\end{array}$ & $\begin{array}{l}\text { Parton Shower } \\
\text { (alternative) }\end{array}$ & $\begin{array}{l}\text { PDF } \\
\text { (alternative) }\end{array}$ & Tune & Cross section \\
\hline$q \bar{q} \rightarrow Z H$ & $\begin{array}{l}\text { POWHEG-BOX v2 [32] } \\
\text { +GOSAM [35] } \\
\text { +MiNLO }[45,46]\end{array}$ & $\begin{array}{l}\text { Pythia } 8 \\
\text { (HeRWig } 7 \text { [47]) }\end{array}$ & $\begin{array}{l}\text { PDF4LHC15NLO [33] } \\
\text { /CTEQ6L1 [36,37] }\end{array}$ & $\begin{array}{l}\text { AZNLO [34] } \\
\text { (A14 [48]) }\end{array}$ & $\begin{array}{l}\text { NNLO }(\mathrm{QCD}) * \\
+\mathrm{NLO}(\mathrm{EW})[38-44]\end{array}$ \\
\hline$g g \rightarrow Z H$ & POWHEG-BOX v2 & $\begin{array}{l}\text { PYTHIA } 8 \\
\text { (HERWIG 7) }\end{array}$ & $\begin{array}{l}\text { PDF4LHC15NLO } \\
\text { /CTEQ6L1 }\end{array}$ & $\begin{array}{l}\text { AZNLO } \\
\text { (A14) }\end{array}$ & NLO+NLL (QCD) $[17,49-51]$ \\
\hline$t \bar{t}$ & POWHEG-BOX v2 & $\begin{array}{l}\text { PYTHIA } 8 \\
\text { (HERWIG 7) }\end{array}$ & $\begin{array}{l}\text { NNPDF3.0NLO [52] } \\
\text { /NNPDF2.3LO }\end{array}$ & A14 & NNLO + NNLL [53] \\
\hline$Z W, Z Z$ & $\begin{array}{l}\text { SHERPA 2.2.1 [54] } \\
\text { (POWHEG-BOX) }\end{array}$ & $\begin{array}{l}\text { SHERPA } \\
\text { (PYTHIA 8) }\end{array}$ & NNPDF3.0NNLO & SHERPA & NLO \\
\hline$Z+$ jets & $\begin{array}{l}\text { SHERPA 2.2.1 } \\
\text { (MG5_AMC) }\end{array}$ & $\begin{array}{l}\text { SHERPA } \\
\text { (PYTHIA 8) }\end{array}$ & $\begin{array}{l}\text { NNPDF3.0NNLO } \\
\text { (NNPDF2.3LO) }\end{array}$ & $\begin{array}{l}\text { SHERPA } \\
\text { (A14) }\end{array}$ & NNLO [55] \\
\hline
\end{tabular}

generated using PowHEG-BOX v2. Backgrounds from single top and multijet production and the contribution from Higgs decays other than $b \bar{b}$ and $c \bar{c}$ are assessed to be negligible and not considered further. The Higgs boson mass is set to $m_{H}=125 \mathrm{GeV}$ and the top-quark mass is set to $172.5 \mathrm{GeV}$.

Events are required to have at least one reconstructed primary vertex. Electron candidates are reconstructed from energy clusters in the electromagnetic calorimeter that are associated with charged-particle tracks reconstructed in the inner detector [56,57]. Muon candidates are reconstructed by combining inner detector tracks with muon spectrometer tracks or energy deposits in the calorimeters consistent with the passage of minimum-ionizing particles [58]. For data recorded in 2015, the single-electron (muon) trigger required a candidate with $p_{\mathrm{T}}>24(20) \mathrm{GeV}$; in 2016 the lepton $p_{\mathrm{T}}$ threshold was raised to $26 \mathrm{GeV}$. Events are required to contain a pair of same-flavor leptons, both satisfying $p_{\mathrm{T}}>7 \mathrm{GeV}$ and $|\eta|<2.5$. At least one lepton must have $p_{\mathrm{T}}>27 \mathrm{GeV}$ and correspond to a lepton that passed the trigger. The two leptons are required to satisfy loose track-isolation criteria with an efficiency greater than $99 \%$. They are required to have opposite charge in dimuon events, but not in dielectron events due to the non-negligible charge misidentification rate of electrons. The invariant mass of the dilepton system is required to be consistent with the mass of the $Z$ boson: $81 \mathrm{GeV}<$ $m_{\ell \ell}<101 \mathrm{GeV}$.

Jets are reconstructed from topological energy clusters in the calorimeters $[59,60]$ using the anti- $k_{t}$ algorithm [61] with a radius parameter of 0.4 implemented in the FASTJET package [62]. The jet energy is corrected using a jet-area- based technique $[63,64]$ and calibrated $[65,66]$ using $p_{\mathrm{T}^{-}}$and $\eta$-dependent correction factors determined from simulation, with residual corrections from internal jet properties. Further corrections from in situ measurements are applied to data. Selected jets must have $p_{\mathrm{T}}>20 \mathrm{GeV}$ and $|\eta|<2.5$. Events are required to contain at least two jets. If a muon is found within a jet, its momentum is added to the selected jet. An overlap removal procedure resolves cases in which the same physical object is reconstructed multiple times, e.g. an electron also reconstructed as a jet.

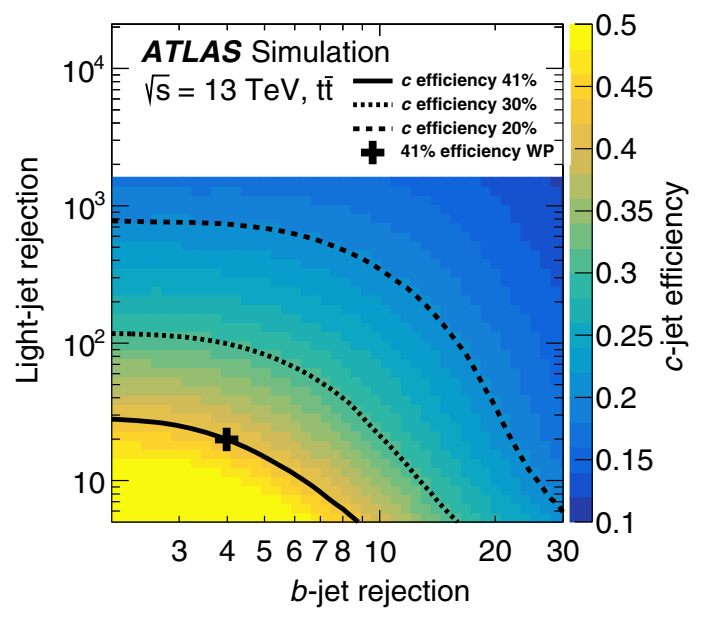

FIG. 1. The $c$-jet-tagging efficiency (colored scale) as a function of the $b$ jet and $l$ jet rejection as obtained from simulated $t \bar{t}$ events. The cross, labeled as working point, WP, denotes the selection criterion used in this analysis. The solid and dotted black lines indicate the contours in rejection space for the fixed $c$-tagging efficiency used in the analysis and two alternatives. 
TABLE II. Breakdown of the relative contributions to the total uncertainty in $\mu$. The statistical uncertainty includes the contribution from the floating $Z+$ jets normalization parameters. The sum in quadrature of the individual components differs from the total uncertainty due to correlations between the components.

\begin{tabular}{lc}
\hline \hline Source & $\sigma / \sigma_{\text {tot }}$ \\
\hline Statistical & $\mathbf{4 9} \%$ \\
Floating $Z+$ jets normalization & $31 \%$ \\
Systematic & $\mathbf{8 7 \%}$ \\
Flavor tagging & $73 \%$ \\
Background modeling & $47 \%$ \\
Lepton, jet and luminosity & $28 \%$ \\
Signal modeling & $28 \%$ \\
MC statistical & $6 \%$ \\
\hline \hline
\end{tabular}

Jets in simulated events are labeled according to the presence of a heavy-flavor hadron with $p_{\mathrm{T}}>5 \mathrm{GeV}$ within $\Delta R=0.3$ from the jet axis. If a $b$ hadron is found the jet is labeled as a $b$ jet. If no $b$ hadron is found, but a $c$ hadron is present, then the jet is labeled as a $c$ jet. Otherwise the jet is labeled as a light-flavor jet ( $l$ jet).

Flavor-tagging algorithms exploit the different lifetimes of $b, c$, and light-flavor hadrons. A $c$-tagging algorithm is used to identify $c$ jets. Charm jets are particularly challenging to tag because $c$ hadrons have shorter lifetimes and decay to fewer charged particles than $b$ hadrons. Boosted decision trees are trained to obtain two multivariate discriminants: to separate $c$ jets from $l$ jets and $c$ jets from $b$ jets. The same variables used for $b$ tagging $[67,68]$ are used. Figure 1 shows the selection criteria applied in the two-dimensional multivariate discriminant space, to obtain an efficiency of $41 \%$ for $c$ jets and rejection factors of 4.0 and 20 for $b$ jets and $l$ jets. The efficiencies are calibrated to data using $b$ quarks from $t \rightarrow W b$ and $c$ quarks from $W \rightarrow c s, c d$ with methods identical to the $b$-tagging algorithms [67]. Statistical uncertainties in the simulation are reduced, by weighting events according to the tagging efficiencies of their jets, parametrized as a function of jet flavor, $p_{\mathrm{T}}, \eta$ and the angular separation between jets, rather than imposing a direct requirement on the $c$-tagging discriminants.

Data are analyzed in four categories with different expected signal purities. The dijet invariant mass, $m_{c \bar{c}}$, constructed using the two highest- $p_{\mathrm{T}}$ jets, is the discriminating variable in each category. Categories are defined using the transverse momentum of the reconstructed $Z$ boson, $p_{\mathrm{T}}^{Z}\left(75 \mathrm{GeV} \leq p_{\mathrm{T}}^{Z}<150 \mathrm{GeV}\right.$ and $\left.p_{\mathrm{T}}^{Z} \geq 150 \mathrm{GeV}\right)$ and the number of $c$ tags amongst the leading jets (either one or two). The $p_{\mathrm{T}}^{Z}$ requirements exploit the harder $p_{\mathrm{T}}^{Z}$ distribution in $Z H$ compared to $Z+$ jets production. Background events are rejected by requiring the angular separation between the two jets constituting the dijet system, $\Delta R_{c \bar{c}}$, to be less than $2.2,1.5$, or 1.3 for events satisfying $75 \leq p_{\mathrm{T}}^{Z}<150 \mathrm{GeV}, 150 \leq p_{\mathrm{T}}^{Z}<200 \mathrm{GeV}$, or $p_{\mathrm{T}}^{Z} \geq 200 \mathrm{GeV}$. The signal acceptance ranges from $0.5 \%$ to $3.4 \%$ depending on the category. A joint binned maximumprofile-likelihood fit to $m_{c \bar{c}}$ in the categories is used to extract the signal yield and the $Z+$ jets background normalization. The fit uses 15 bins in each category within the range of $50 \mathrm{GeV}<m_{c \bar{c}}<200 \mathrm{GeV}$, with a bin width of $10 \mathrm{GeV}$. The parameter of interest, $\mu$, common to all categories, is the signal strength, defined as the ratio of the measured signal yield to the SM prediction.

Systematic uncertainties affecting the signal and background predictions include theoretical uncertainties in the signal and background modeling and experimental uncertainties. Table II shows their relative impact on the fitted value of $\mu$. Uncertainties in the $m_{c \bar{c}}$ shape of the backgrounds are assessed by comparisons between nominal and alternative event generators as indicated in Table I.

Systematic uncertainties are incorporated within the statistical model through nuisance parameters that modify the shape and/or normalization of the distributions. Statistical uncertainties in the simulation samples are accounted for. The $Z+$ jets background is normalized from the data through the inclusion of an unconstrained normalization parameter for each category. The fitted

TABLE III. Postfit yields for the signal and background processes in each category from the profile likelihood fit. Uncertainties include statistical and systematic contributions. The prefit SM expected $Z H(c \bar{c})$ signal yields are indicated in parenthesis.

\begin{tabular}{lcccc}
\hline \hline \multirow{2}{*}{ Sample } & \multicolumn{4}{c}{ Yield, $50 \mathrm{GeV}<m_{c \bar{c}}<200 \mathrm{GeV}$} \\
\cline { 2 - 4 } & \multicolumn{2}{c}{$1 c$ tag } & & $2 c$ tags \\
\cline { 2 - 5 } & $75 \leq p_{\mathrm{T}}^{Z}<150 \mathrm{GeV}$ & $p_{\mathrm{T}}^{Z} \geq 150 \mathrm{GeV}$ & $5320 \pm 100$ & $1280 \pm 40$ \\
\hline$Z+$ jets & $69400 \pm 500$ & $15650 \pm 180$ & $53 \pm 13$ & $20 \pm 5$ \\
$Z W$ & $750 \pm 130$ & $290 \pm 50$ & $55 \pm 18$ & $26 \pm 8$ \\
$Z Z$ & $490 \pm 70$ & $180 \pm 28$ & $240 \pm 40$ & $13 \pm 6$ \\
$t \bar{t}$ & $2020 \pm 280$ & $130 \pm 50$ & $4.1 \pm 0.4$ & $2.7 \pm 0.2$ \\
$Z H(b \bar{b})$ & $32 \pm 2$ & $19.5 \pm 1.5$ & $-30 \pm 40(0.7)$ & $-20 \pm 29(0.5)$ \\
$Z H(c \bar{c})(\mathrm{SM})$ & $-143 \pm 170(2.4)$ & $-84 \pm 100(1.4)$ & $5650 \pm 80$ & $1320 \pm 40$ \\
Total & $72500 \pm 320$ & $16180 \pm 140$ & 5648 & 1320 \\
Data & 72504 & 16181 & & \\
\hline \hline
\end{tabular}




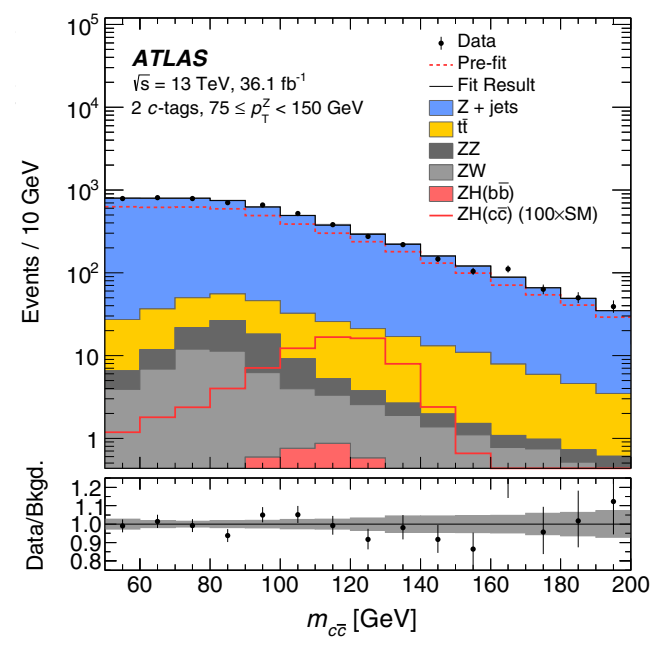

(a)

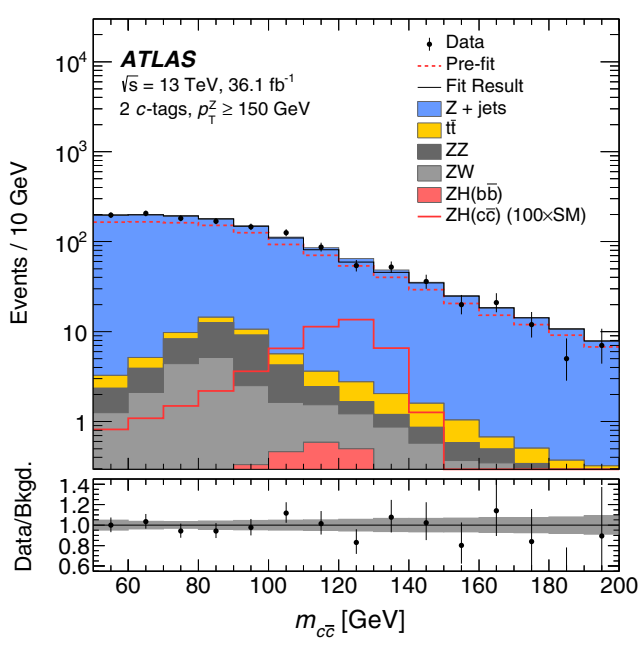

(b)

FIG. 2. Observed and predicted $m_{c \bar{c}}$ distributions in the $2 c$-tag analysis categories. The expected signal is scaled by a factor of 100 . Backgrounds are corrected to the results of the fit to the data. The predicted background from the simulation is shown as red dashed histograms. The ratios of the data to the fitted background are shown in the lower panels. The error bands indicate the sum in quadrature of the statistical and systematic uncertainties in the background prediction.

normalization parameters range between 1.13 and 1.30. All other background normalization factors are correlated between categories, with acceptance uncertainties of order $10 \%$ to account for relative variations between categories.

The dominant contributions to the uncertainty in $\mu$ are the efficiency of the tagging algorithms, the jet energy scale and resolution, and the background modeling. The largest uncertainty is due to the normalization of the dominant $Z+$ jets background. The typical uncertainty in the tagging efficiency is $25 \%$ for $c$ jets, $5 \%$ for $b$ jets, and $20 \%$ for $l$ jets.

Table III shows the fitted signal and background yields. The $m_{c \bar{c}}$ distributions in the $2 c$ tag categories are shown in Fig. 2 with the background shapes and normalizations according to the result of the fit. Good agreement is observed between the postfit shapes of the distributions and the data.

The analysis procedure is validated by measuring the yield of $Z V$ production, where $V$ denotes a $W$ or $Z$ boson, with the same event selection. The fraction of the $Z Z$ yield from $Z \rightarrow c \bar{c}$ decays is $\sim 55 \%$ (20\%) in the $2 c$ tag (1 $c$ tag) category, while the fraction of the $Z W$ yield from $W \rightarrow c s$, $c d$ is $\sim 65 \%$ for both the 2 and $1 c$ tag categories. Contributions of Higgs boson decays to $c \bar{c}$ and $b \bar{b}$ are treated as background and constrained to the SM predictions within its theoretical uncertainties. The diboson signal strength is measured to be $\mu_{Z V}=0.6_{-0.4}^{+0.5}$ with an observed (expected) significance of 1.4 (2.2) standard deviations.

The best-fit value for the $Z H(c \bar{c})$ signal strength is $\mu_{Z H}=-69 \pm 101$. By assuming a signal with the kinematics of the SM Higgs boson, model-dependent corrections are made to extrapolate to the inclusive phase space. Hence, an upper limit on $\sigma(p p \rightarrow Z H) \times \mathcal{B}(H \rightarrow c \bar{c})$ is computed using a modified frequentist $\mathrm{CL}_{\mathrm{s}}$ method $[69,70]$ with the profile likelihood ratio as the test statistic. The observed (expected) upper limit is found to be $2.7\left(3.9_{-1.1}^{+2.1}\right)$ $\mathrm{pb}$ at the 95\% C.L. This corresponds to an observed (expected) upper limit on $\mu$ at the $95 \%$ C.L. of 110 $\left(150_{-40}^{+80}\right)$. The uncertainties in the expected limits correspond to the $\pm 1 \sigma$ interval of background-only pseudoexperiments. With the current sensitivity, the result depends weakly on the assumption of the SM rate for $H \rightarrow b \bar{b}$. The observed limit remains within $5 \%$ of the nominal value when the assumed value for normalization of the $Z H(b \bar{b})$ background is varied from zero to twice the SM prediction.

A search for the decay of the Higgs boson to charm quarks has been performed using $36.1 \mathrm{fb}^{-1}$ of data collected with the ATLAS detector in $p p$ collisions at $\sqrt{s}=$ $13 \mathrm{TeV}$ at the LHC. No significant excess of $Z H(c \bar{c})$ production is observed over the SM background expectation. The observed upper limit on $\sigma(p p \rightarrow Z H) \times \mathcal{B}(H \rightarrow$ $c \bar{c})$ is $2.7 \mathrm{pb}$ at the $95 \%$ C.L. The corresponding expected upper limit is $3.9_{-1.1}^{+2.1} \mathrm{pb}$. This is the most stringent limit to date in direct searches for the inclusive decay of the Higgs boson to charm quarks.

We thank CERN for the very successful operation of the LHC, as well as the support staff from our institutions without whom ATLAS could not be operated efficiently. We acknowledge the support of ANPCyT, Argentina; YerPhI, Armenia; ARC, Australia; BMWFW and FWF, Austria; ANAS, Azerbaijan; SSTC, Belarus; CNPq and FAPESP, Brazil; NSERC, NRC and CFI, Canada; CERN; CONICYT, Chile; CAS, MOST and NSFC, China; COLCIENCIAS, Colombia; MSMT CR, MPO CR and VSC CR, Czech Republic; DNRF and DNSRC, Denmark; IN2P3-CNRS, CEA-DRF/IRFU, France; SRNSFG, 
Georgia; BMBF, HGF, and MPG, Germany; GSRT, Greece; RGC, Hong Kong SAR, China; ISF, I-CORE and Benoziyo Center, Israel; INFN, Italy; MEXT and JSPS, Japan; CNRST, Morocco; NWO, Netherlands; RCN, Norway; MNiSW and NCN, Poland; FCT, Portugal; MNE/IFA, Romania; MES of Russia and NRC KI, Russian Federation; JINR; MESTD, Serbia; MSSR, Slovakia; ARRS and MIZŠ, Slovenia; DST/NRF, South Africa; MINECO, Spain; SRC and Wallenberg Foundation, Sweden; SERI, SNSF and Cantons of Bern and Geneva, Switzerland; MOST, Taiwan; TAEK, Turkey; STFC, United Kingdom; DOE and NSF, United States of America. In addition, individual groups and members have received support from BCKDF, the Canada Council, CANARIE, CRC, Compute Canada, FQRNT, and the Ontario Innovation Trust, Canada; EPLANET, ERC, ERDF, FP7, Horizon 2020 and Marie Skłodowska-Curie Actions, European Union; Investissements d'Avenir Labex and Idex, ANR, Région Auvergne and Fondation Partager le Savoir, France; DFG and AvH Foundation, Germany; Herakleitos, Thales and Aristeia programmes co-financed by EU-ESF and the Greek NSRF; BSF, GIF and Minerva, Israel; BRF, Norway; CERCA Programme Generalitat de Catalunya, Generalitat Valenciana, Spain; the Royal Society and Leverhulme Trust, United Kingdom. The crucial computing support from all WLCG partners is acknowledged gratefully, in particular from CERN, the ATLAS Tier-1 facilities at TRIUMF (Canada), NDGF (Denmark, Norway, Sweden), CC-IN2P3 (France), KIT/ GridKA (Germany), INFN-CNAF (Italy), NL-T1 (Netherlands), PIC (Spain), ASGC (Taiwan), RAL (UK) and BNL (USA), the Tier-2 facilities worldwide and large non-WLCG resource providers. Major contributors of computing resources are listed in Ref. [71].

[1] ATLAS Collaboration, Observation of a new particle in the search for the Standard Model Higgs boson with the ATLAS detector at the LHC, Phys. Lett. B 716, 1 (2012).

[2] CMS Collaboration, Observation of a new boson with mass near $125 \mathrm{GeV}$ in pp collisions at $\sqrt{s}=7$ and $8 \mathrm{TeV}$, J. High Energy Phys. 06 (2013) 081.

[3] L. Evans and P. Bryant, LHC machine, J. Instrum. 3, S08001 (2008).

[4] ATLAS Collaboration, Measurements of the Higgs boson production and decay rates and coupling strengths using $p p$ collision data at $\sqrt{s}=7$ and $8 \mathrm{TeV}$ in the ATLAS experiment, Eur. Phys. J. C 76, 6 (2016).

[5] CMS Collaboration, Precise determination of the mass of the Higgs boson and tests of compatibility of its couplings with the standard model predictions using proton collisions at 7 and 8 TeV, Eur. Phys. J. C 75, 212 (2015).

[6] CMS Collaboration, Study of the Mass and Spin-Parity of the Higgs Boson Candidate Via Its Decays to Z Boson Pairs, Phys. Rev. Lett. 110, 081803 (2013).
[7] ATLAS Collaboration, Evidence for the spin-0 nature of the Higgs boson using ATLAS data, Phys. Lett. B 726, 120 (2013).

[8] CMS Collaboration, Constraints on the spin-parity and anomalous HVV couplings of the Higgs boson in proton collisions at 7 and 8 TeV, Phys. Rev. D 92, 012004 (2015).

[9] ATLAS and CMS Collaborations, Measurements of the Higgs boson production and decay rates and constraints on its couplings from a combined ATLAS and CMS analysis of the LHC $p p$ collision data at $\sqrt{s}=7$ and $8 \mathrm{TeV}$, J. High Energy Phys. 08 (2016) 045.

[10] ATLAS and CMS Collaborations, Combined Measurement of the Higgs Boson Mass in $p p$ Collisions at $\sqrt{s}=7$ and $8 \mathrm{TeV}$ with the ATLAS and CMS Experiments, Phys. Rev. Lett. 114, 191803 (2015).

[11] ATLAS Collaboration, Evidence for the associated production of the Higgs boson and a top quark pair with the ATLAS detector, Phys. Rev. D 97, 072003 (2017).

[12] ATLAS Collaboration, Evidence for the $H \rightarrow b \bar{b}$ decay with the ATLAS detector, J. High Energy Phys. 12 (2017) 024.

[13] CMS Collaboration, Evidence for the Higgs boson decay to a bottom quark-antiquark pair, Phys. Lett. B 780, 501 (2018).

[14] G. Perez, Y. Soreq, E. Stamou, and K. Tobioka, Constraining the charm Yukawa and Higgs-quark coupling universality, Phys. Rev. D 92, 033016 (2015).

[15] G. Perez, Y. Soreq, E. Stamou, and K. Tobioka, Prospects for measuring the Higgs boson coupling to light quarks, Phys. Rev. D 93, 013001 (2016).

[16] A. Djouadi, J. Kalinowski, and M. Spira, HDECAY: A Program for Higgs boson decays in the standard model and its supersymmetric extension, Comput. Phys. Commun. 108, 56 (1998).

[17] D. de Florian et al., Handbook of LHC Higgs cross sections: 4. Deciphering the nature of the Higgs sector, arXiv: 1610.07922.

[18] G. T. Bodwin, F. Petriello, S. Stoynev, and M. Velasco, Higgs boson decays to quarkonia and the $H \bar{c} c$ coupling, Phys. Rev. D 88, 053003 (2013).

[19] A. L. Kagan, G. Perez, F. Petriello, Y. Soreq, S. Stoynev, and J. Zupan, Exclusive Window onto Higgs Yukawa Couplings, Phys. Rev. Lett. 114, 101802 (2015).

[20] ATLAS Collaboration, Search for Higgs and Z Boson Decays to $J / \psi \gamma$ and $\Upsilon(n S) \gamma$ with the ATLAS Detector, Phys. Rev. Lett. 114, 121801 (2015).

[21] ATLAS Collaboration, Search for Higgs and $Z$ Boson Decays to $\phi \gamma$ with the ATLAS Detector, Phys. Rev. Lett. 117, 111802 (2016).

[22] C. Delaunay, T. Golling, G. Perez, and Y. Soreq, Enhanced Higgs boson coupling to charm pairs, Phys. Rev. D 89, 033014 (2014).

[23] ATLAS Collaboration, The ATLAS Experiment at the CERN Large Hadron Collider, J. Instrum. 3, S08003 (2008).

[24] ATLAS uses a right-handed coordinate system with its origin at the nominal interaction point (IP) in the center of the detector and the $z$ axis along the beam pipe. The $x$ axis points from the IP to the center of the LHC ring, and the $y$ axis points upwards. Cylindrical coordinates $(r, \phi)$ are used in the transverse plane, $\phi$ being the azimuthal angle around the $z$ axis. The pseudorapidity is defined in terms of 
the polar angle $\theta$ as $\eta=-\ln \tan (\theta / 2)$. Angular distance is measured in units of $\Delta R \equiv \sqrt{(\Delta \eta)^{2}+(\Delta \phi)^{2}}$.

[25] ATLAS Collaboration, ATLAS insertable B-layer technical design report, Report No. ATLAS-TDR-19, 2010, https:// cds.cern.ch/record/1291633; ATLAS insertable B-layer technical design report addendum, Report No. ATLASTDR-19-ADD-1, 2012, https://cds.cern.ch/record/1451888.

[26] ATLAS Collaboration, Luminosity determination in $p p$ collisions at $\sqrt{s}=8 \mathrm{TeV}$ using the ATLAS detector at the LHC, Eur. Phys. J. C 76, 653 (2016).

[27] ATLAS Collaboration, The ATLAS simulation infrastructure, Eur. Phys. J. C 70, 823 (2010).

[28] S. Agostinelli et al. (GEANT4 Collaboration), GEANT4A simulation toolkit, Nucl. Instrum. Methods Phys. Res., Sect. A 506, 250 (2003).

[29] J. Alwall, R. Frederix, S. Frixione, V. Hirschi, F. Maltoni, O. Mattelaer, H.-S. Shao, T. Stelzer, P. Torrielli, and M. Zaro, The automated computation of tree-level and next-toleading order differential cross sections, and their matching to parton shower simulations, J. High Energy Phys. 07 (2014) 079.

[30] T. Sjöstrand, S. Mrenna, and P.Z. Skands, A brief introduction to PYTHIA 8.1, Comput. Phys. Commun. 178, 852 (2008).

[31] D. J. Lange, The EvtGen particle decay simulation package, Nucl. Instrum. Methods Phys. Res., Sect. A 462, 152 (2001).

[32] S. Alioli, P. Nason, C. Oleari, and E. Re, A general framework for implementing NLO calculations in shower Monte Carlo programs: The POWHEG BOX, J. High Energy Phys. 06 (2010) 043.

[33] J. Butterworth et al., PDF4LHC recommendations for LHC Run II, J. Phys. G 43, 023001 (2016).

[34] ATLAS Collaboration, Measurement of the $Z / \gamma^{*}$ boson transverse momentum distribution in $p p$ collisions at $\sqrt{s}=$ $7 \mathrm{TeV}$ with the ATLAS detector, J. High Energy Phys. 09 (2014) 145.

[35] G. Cullen, N. Greiner, G. Heinrich, G. Luisoni, P. Mastrolia, G. Ossola, T. Reiter, and F. Tramontano, Automated oneloop calculations with GoSAM, Eur. Phys. J. C 72, 1889 (2012).

[36] J. Pumplin, D. R. Stump, J. Huston, H.-L. Lai, P. Nadolsky, and W.-K. Tung, New generation of parton distributions with uncertainties from global QCD analysis, J. High Energy Phys. 07 (2002) 012.

[37] P. M. Nadolsky, H.-L. Lai, Q.-H. Cao, J. Huston, J. Pumplin, D. Stump, W.-K. Tung, and C.-P. Yuan, Implications of CTEQ global analysis for collider observables, Phys. Rev. D 78, 013004 (2008).

[38] M. L. Ciccolini, S. Dittmaier, and M. Krämer, Electroweak radiative corrections to associated $\mathrm{WH}$ and $\mathrm{ZH}$ production at hadron colliders, Phys. Rev. D 68, 073003 (2003).

[39] O. Brein, A. Djouadi, and R. Harlander, NNLO QCD corrections to the Higgs-strahlung processes at hadron colliders, Phys. Lett. B 579, 149 (2004).

[40] G. Ferrera, M. Grazzini, and F. Tramontano, Associated WH Production at Hadron Colliders: A Fully Exclusive QCD Calculation at NNLO, Phys. Rev. Lett. 107, 152003 (2011).
[41] O. Brein, R. Harlander, M. Wiesemann, and T. Zirke, Top-quark mediated effects in hadronic Higgs-Strahlung, Eur. Phys. J. C 72, 1868 (2012).

[42] G. Ferrera, M. Grazzini, and F. Tramontano, Higher-order QCD effects for associated WH production and decay at the LHC, J. High Energy Phys. 04 (2014) 039.

[43] G. Ferrera, M. Grazzini, and F. Tramontano, Associated ZH production at hadron colliders: the fully differential NNLO QCD calculation, Phys. Lett. B 740, 51 (2015).

[44] J. M. Campbell, R. K. Ellis, and C. Williams, Associated production of a Higgs boson at NNLO, J. High Energy Phys. 06 (2016) 179.

[45] K. Hamilton, P. Nason, and G. Zanderighi, MINLO: multiscale improved NLO, J. High Energy Phys. 10 (2012) 155.

[46] G. Luisoni, P. Nason, C. Oleari, and F. Tramontano, $H W^{ \pm} / \mathrm{HZ}+0$ and 1 jet at NLO with the POWHEG BOX interfaced to GoSAM and their merging within MiNLO, J. High Energy Phys. 10 (2013) 083.

[47] J. Bellm et al., Herwig 7.0/Herwig ++3.0 release note, Eur. Phys. J. C 76, 196 (2016).

[48] ATLAS Collaboration, ATLAS Pythia 8 tunes to $7 \mathrm{TeV}$ data, Report No. ATL-PHYS-PUB-2014-021, 2014, https:// cds.cern.ch/record/1966419.

[49] L. Altenkamp, S. Dittmaier, R. V. Harlander, H. Rzehak, and T. J. E. Zirke, Gluon-induced Higgs-strahlung at next-toleading order QCD, J. High Energy Phys. 02 (2013) 078.

[50] B. Hespel, F. Maltoni, and E. Vryonidou, Higgs and $\mathrm{Z}$ boson associated production via gluon fusion in the SM and the 2HDM, J. High Energy Phys. 06 (2015) 065.

[51] L. Altenkamp, S. Dittmaier, R. V. Harlander, H. Rzehak, and T. J. Zirke, Gluon-induced Higgs-strahlung at next-toleading order QCD, J. High Energy Phys. 02 (2013) 078.

[52] R. D. Ball et al., Parton distributions with LHC data, Nucl. Phys. B867, 244 (2013).

[53] M. Czakon and A. Mitov, Top ++ : A program for the calculation of the top-pair cross section at hadron colliders, Comput. Phys. Commun. 185, 2930 (2014).

[54] T. Gleisberg, S. Höche, F. Krauss, M. Schönherr, S. Schumann, F. Siegert, and J. Winter, Event generation with SHERPA 1.1, J. High Energy Phys. 02 (2009) 007.

[55] S. Catani, L. Cieri, G. Ferrera, D. de Florian, and M. Grazzini, Vector Boson Production at Hadron Colliders: A Fully Exclusive QCD Calculation at Next-to-Next-toLeading Order, Phys. Rev. Lett. 103, 082001 (2009).

[56] ATLAS Collaboration, Electron efficiency measurements with the ATLAS detector using 2012 LHC proton-proton collision data, Eur. Phys. J. C 77, 195 (2017).

[57] ATLAS Collaboration, Electron efficiency measurements with the ATLAS detector using the 2015 LHC protonproton collision data, Report No. ATLAS-CONF-2016-024, 2016, https://cds.cern.ch/record/2157687.

[58] ATLAS Collaboration, Muon reconstruction performance of the ATLAS detector in proton-proton collision data at $\sqrt{s}=13 \mathrm{TeV}$, Eur. Phys. J. C 76, 292 (2016).

[59] ATLAS Collaboration, Topological cell clustering in the ATLAS calorimeters and its performance in LHC Run 1, Eur. Phys. J. C 77, 490 (2017).

[60] ATLAS Collaboration, Properties of jets and inputs to jet reconstruction and calibration with the ATLAS detector 
using proton-proton collisions at $\sqrt{s}=13 \mathrm{TeV}, 2015$, https://cds.cern.ch/record/2044564.

[61] M. Cacciari, G. P. Salam, and G. Soyez, The anti- $k_{t}$ jet clustering algorithm, J. High Energy Phys. 04 (2008) 063.

[62] M. Cacciari, G. P. Salam, and G. Soyez, FAstJet User Manual, Eur. Phys. J. C 72, 1896 (2012).

[63] M. Cacciari and G. P. Salam, Pileup subtraction using jet areas, Phys. Lett. B 659, 119 (2008).

[64] ATLAS Collaboration, Performance of pile-up mitigation techniques for jets in $p p$ collisions at $\sqrt{s}=8 \mathrm{TeV}$ using the ATLAS detector, Eur. Phys. J. C 76, 581 (2016).

[65] ATLAS Collaboration, Jet energy measurement with the ATLAS detector in proton-proton collisions at $\sqrt{s}=$ 7 TeV, Eur. Phys. J. C 73, 2304 (2013).

[66] ATLAS Collaboration, Jet energy scale measurements and their systematic uncertainties in proton-proton collisions at $\sqrt{s}=13 \mathrm{TeV}$ with the ATLAS detector, Phys. Rev. D 96, 072002 (2017).
[67] ATLAS Collaboration, Performance of $b$ jet identification in the ATLAS experiment, J. Instrum. 11, P04008 (2016).

[68] ATLAS Collaboration, Optimization of the ATLAS $b$-tagging performance for the 2016 LHC Run, Report No. ATL-PHYS-PUB-2016-012, 2016, https://cds.cern.ch/ record/2160731.

[69] G. Cowan, K. Cranmer, E. Gross, and O. Vitells, Asymptotic formulae for likelihood-based tests of new physics, Eur. Phys. J. C 71, 1554 (2011).

[70] A. L. Read, Presentation of search results: The CLS technique, J. Phys. G 28, 2693 (2002).

[71] ATLAS Collaboration, ATLAS Computing Acknowledgements 2016-2017, Report No. ATL-GEN-PUB-2016-002, https://cds.cern.ch/record/2202407.

M. Aaboud, ${ }^{137 \mathrm{~d}}$ G. Aad ${ }^{88}$ B. Abbott, ${ }^{115}$ O. Abdinov, ${ }^{12, \mathrm{a}}$ B. Abeloos, ${ }^{119}$ S. H. Abidi, ${ }^{161}$ O. S. AbouZeid, ${ }^{139}$ N. L. Abraham, ${ }^{151}$ H. Abramowicz, ${ }^{155}$ H. Abreu, ${ }^{154}$ Y. Abulaiti, ${ }^{6}$ B. S. Acharya,${ }^{167 a, 167 b, b}$ S. Adachi, ${ }^{157}$ L. Adamczyk,${ }^{41 a}$ J. Adelman ${ }^{110}$ M. Adersberger, ${ }^{102}$ T. Adye, ${ }^{133}$ A. A. Affolder, ${ }^{139}$ Y. Afik, ${ }^{154}$ C. Agheorghiesei, ${ }^{28 c}$ J. A. Aguilar-Saavedra, ${ }^{128 a, 128 f}$ S. P. Ahlen, ${ }^{24}$ F. Ahmadov, ${ }^{68, c}$ G. Aielli, ${ }^{135 a, 135 b}$ S. Akatsuka, ${ }^{71}$ T. P. A. Åkesson, ${ }^{84}$ E. Akilli, ${ }^{52}$ A. V. Akimov, ${ }^{98}$ G. L. Alberghi, ${ }^{22 a, 22 b}$ J. Albert, ${ }^{172}$ P. Albicocco,${ }^{50}$ M. J. Alconada Verzini, ${ }^{74}$ S. Alderweireldt, ${ }^{108}$ M. Aleksa ${ }^{32}$ I. N. Aleksandrov, ${ }^{68}$ C. Alexa, ${ }^{28 b}$ G. Alexander, ${ }^{155}$ T. Alexopoulos, ${ }^{10}$ M. Alhroob, ${ }^{115}$ B. Ali, ${ }^{130}$ M. Aliev, ${ }^{76 a, 76 b}$ G. Alimonti, ${ }^{94 a}$ J. Alison, ${ }^{33}$ S. P. Alkire,${ }^{38}$ C. Allaire, ${ }^{119}$ B. M. M. Allbrooke,${ }^{151}$ B. W. Allen, ${ }^{118}$ P. P. Allport,,${ }^{19}$ A. Aloisio, ${ }^{106 a, 106 b}$ A. Alonso, ${ }^{39}$ F. Alonso, ${ }^{74}$ C. Alpigiani, ${ }^{140}$ A. A. Alshehri, ${ }^{56}$ M. I. Alstaty, ${ }^{88}$ B. Alvarez Gonzalez, ${ }^{32}$ D. Álvarez Piqueras, ${ }^{170}$ M. G. Alviggi, ${ }^{106 a, 106 b}$ B. T. Amadio, ${ }^{16}$ Y. Amaral Coutinho, ${ }^{26 a}$ L. Ambroz, ${ }^{122}$ C. Amelung, ${ }^{25}$ D. Amidei, ${ }^{92}$ S. P. Amor Dos Santos, ${ }^{128 a, 128 c}$ S. Amoroso, ${ }^{32}$ C. Anastopoulos ${ }^{141}$ L. S. Ancu, ${ }^{52}$ N. Andari, ${ }^{19}$ T. Andeen, ${ }^{11}$ C. F. Anders, ${ }^{60 \mathrm{~b}}$ J. K. Anders, ${ }^{18}$ K. J. Anderson, ${ }^{33}$ A. Andreazza, ${ }^{94 a}, 94 \mathrm{~b}$ V. Andrei, ${ }^{60 \mathrm{a}}$ S. Angelidakis, ${ }^{37}$ I. Angelozzi, ${ }^{109}$ A. Angerami, ${ }^{38}$ A. V. Anisenkov, ${ }^{11, d}$ A. Annovi, ${ }^{126 a}$ C. Antel,${ }^{60 a}$ M. Antonelli, ${ }^{50}$ A. Antonov, ${ }^{100, a}$ D. J. Antrim, ${ }^{166}$ F. Anulli, ${ }^{134 a}$ M. Aoki ${ }^{69}$ L. Aperio Bella ${ }^{32}$ G. Arabidze, ${ }^{93}$ Y. Arai, ${ }^{69}$ J. P. Araque, ${ }^{128 a}$ V. Araujo Ferraz, ${ }^{26 a}$ R. Araujo Pereira, ${ }^{26 a}$ A. T. H. Arce, ${ }^{48}$ R. E. Ardell, ${ }^{80}$ F. A. Arduh, ${ }^{74}$ J-F. Arguin, ${ }^{97}$ S. Argyropoulos, ${ }^{66}$ A. J. Armbruster,${ }^{32}$ L. J. Armitage, ${ }^{79}$ O. Arnaez ${ }^{161}$ H. Arnold,${ }^{109}$ M. Arratia, ${ }^{30}$ O. Arslan, ${ }^{23}$ A. Artamonov, ${ }^{99, a}$ G. Artoni, ${ }^{122}$ S. Artz, ${ }^{86}$ S. Asai, ${ }^{157}$ N. Asbah, ${ }^{45}$ A. Ashkenazi, ${ }^{155}$ L. Asquith, ${ }^{151}$ K. Assamagan, ${ }^{27}$ R. Astalos, ${ }^{146 a}$ R. J. Atkin, ${ }^{147 a}$ M. Atkinson, ${ }^{169}$ N. B. Atlay, ${ }^{143}$ K. Augsten, ${ }^{130}$ G. Avolio, ${ }^{32}$ R. Avramidou, ${ }^{36 a}$ B. Axen, ${ }^{16}$ M. K. Ayoub, ${ }^{35 a}$ G. Azuelos, ${ }^{97, e}$ A. E. Baas, ${ }^{60 a}$ M. J. Baca, ${ }^{19}$ H. Bachacou, ${ }^{138}$ K. Bachas, ${ }^{76 a, 76 b}$ M. Backes, ${ }^{122}$ P. Bagnaia, ${ }^{134 a, 134 b}$ M. Bahmani, ${ }^{42}$ H. Bahrasemani, ${ }^{144}$ J. T. Baines, ${ }^{133}$ M. Bajic ${ }^{39}$ O. K. Baker, ${ }^{179}$ P. J. Bakker, ${ }^{109}$ D. Bakshi Gupta ${ }^{82}$ E. M. Baldin, ${ }^{11, d}$ P. Balek, ${ }^{175}$ F. Balli, ${ }^{138}$ W. K. Balunas, ${ }^{124}$ E. Banas, ${ }^{42}$ A. Bandyopadhyay, ${ }^{23}$ Sw. Banerjee, ${ }^{176, f}$ A. A. E. Bannoura,${ }^{177}$ L. Barak, ${ }^{155}$ E. L. Barberio, ${ }^{91}$ D. Barberis, ${ }^{53 a, 53 b}$ M. Barbero, ${ }^{88}$ T. Barillari, ${ }^{103}$ M-S Barisits, ${ }^{65}$ J. T. Barkeloo, ${ }^{118}$ T. Barklow, ${ }^{145}$ N. Barlow, ${ }^{30}$ R. Barnea,${ }^{154}$ S. L. Barnes, ${ }^{36 c}$ B. M. Barnett, ${ }^{133}$ R. M. Barnett, ${ }^{16}$ Z. Barnovska-Blenessy, ${ }^{36 a}$ A. Baroncelli, ${ }^{136 a}$ G. Barone, ${ }^{25}$ A. J. Barr, ${ }^{122}$ L. Barranco Navarro, ${ }^{170}$ F. Barreiro, ${ }^{85}$ J. Barreiro Guimarães da Costa, ${ }^{35 a}$ R. Bartoldus, ${ }^{145}$ A. E. Barton, ${ }^{75}$ P. Bartos, ${ }^{146 a}$ A. Basalaev, ${ }^{125}$ A. Bassalat, ${ }^{119, \mathrm{~g}}$ R. L. Bates,${ }^{56}$ S. J. Batista, ${ }^{161}$ J. R. Batley ${ }^{30}$ M. Battaglia, ${ }^{139}$ M. Bauce, ${ }^{134 a, 134 b}$ F. Bauer, ${ }^{138}$ K. T. Bauer, ${ }^{166}$ H. S. Bawa, ${ }^{145, h}$ J. B. Beacham, ${ }^{113}$ M. D. Beattie, ${ }^{75}$ T. Beau, ${ }^{83}$ P. H. Beauchemin, ${ }^{165}$ P. Bechtle, ${ }^{23}$ H. P. Beck, ${ }^{18, i}$ H. C. Beck, ${ }^{58}$ K. Becker,${ }^{122}$ M. Becker, ${ }^{86}$ C. Becot, ${ }^{112}$ A. J. Beddall, ${ }^{20 \mathrm{e}}$ A. Beddall, ${ }^{20 \mathrm{~b}}$ V. A. Bednyakov,${ }^{68}$ M. Bedognetti, ${ }^{109}$ C. P. Bee, ${ }^{150}$ T. A. Beermann, ${ }^{32}$ M. Begalli, ${ }^{26 a}$ M. Begel,${ }^{27}$ A. Behera, ${ }^{150}$ J. K. Behr, ${ }^{45}$ A. S. Bell, ${ }^{81}$ G. Bella, ${ }^{155}$ L. Bellagamba, ${ }^{22 a}$ A. Bellerive, ${ }^{31}$ M. Bellomo, ${ }^{154}$ K. Belotskiy, ${ }^{100}$ N. L. Belyaev, ${ }^{100}$ O. Benary, ${ }^{155, a}$ D. Benchekroun, ${ }^{137 a}$ M. Bender, ${ }^{102}$ N. Benekos, ${ }^{10}$ Y. Benhammou, ${ }^{155}$ E. Benhar Noccioli, ${ }^{179}$ J. Benitez, ${ }^{66}$ D. P. Benjamin,${ }^{48}$ M. Benoit, ${ }^{52}$ J. R. Bensinger, ${ }^{25}$ S. Bentvelsen, ${ }^{109}$ L. Beresford, ${ }^{122}$ M. Beretta, ${ }^{50}$ D. Berge, ${ }^{45}$ E. Bergeaas Kuutmann, ${ }^{168}$ N. Berger, ${ }^{5}$ L. J. Bergsten, ${ }^{25}$ J. Beringer, ${ }^{16}$ S. Berlendis, ${ }^{57}$ N. R. Bernard, ${ }^{89}$ G. Bernardi, ${ }^{83}$ C. Bernius, ${ }^{145}$ F. U. Bernlochner, ${ }^{23}$ 
T. Berry, ${ }^{80}$ P. Berta, ${ }^{86}$ C. Bertella, ${ }^{35 a}$ G. Bertoli, ${ }^{148 a, 148 b}$ I. A. Bertram, ${ }^{75}$ C. Bertsche,${ }^{45}$ G. J. Besjes,${ }^{39}$ O. Bessidskaia Bylund, ${ }^{148 a, 148 b}$ M. Bessner, ${ }^{45}$ N. Besson, ${ }^{138}$ A. Bethani, ${ }^{87}$ S. Bethke, ${ }^{103}$ A. Betti, ${ }^{23}$ A. J. Bevan, ${ }^{79}$ J. Beyer, ${ }^{103}$ R. M. Bianchi, ${ }^{127}$ O. Biebel,${ }^{102}$ D. Biedermann, ${ }^{17}$ R. Bielski, ${ }^{87}$ K. Bierwagen, ${ }^{86}$ N. V. Biesuz, ${ }^{126 a, 126 b}$ M. Biglietti, ${ }^{136 a}$ T. R. V. Billoud ${ }^{97}$ M. Bindi, ${ }^{58}$ A. Bingul, ${ }^{20 \mathrm{~b}}$ C. Bini, ${ }^{134 \mathrm{a}, 134 \mathrm{~b}}$ S. Biondi, ${ }^{22 \mathrm{a}, 22 \mathrm{~b}}$ T. Bisanz ${ }^{58}$ C. Bittrich, ${ }^{47}$ D. M. Bjergaard, ${ }^{48}$ J. E. Black, ${ }^{145}$ K. M. Black, ${ }^{24}$ R. E. Blair, ${ }^{6}$ T. Blazek, ${ }^{146 a}$ I. Bloch, ${ }^{45}$ C. Blocker, ${ }^{25}$ A. Blue ${ }^{56}$ U. Blumenschein, ${ }^{79}$ Dr. Blunier, ${ }^{34 a}$ G. J. Bobbink, ${ }^{109}$ V. S. Bobrovnikov,${ }^{11, d}$ S. S. Bocchetta, ${ }^{84}$ A. Bocci, ${ }^{48}$ C. Bock, ${ }^{102}$ D. Boerner,${ }^{177}$ D. Bogavac, ${ }^{102}$ A. G. Bogdanchikov, ${ }^{111}$ C. Bohm, ${ }^{148 a}$ V. Boisvert,${ }^{80}$ P. Bokan, ${ }^{168, j}$ T. Bold, ${ }^{41 a}$ A. S. Boldyrev, ${ }^{101}$ A. E. Bolz, ${ }^{60 b}$ M. Bomben,${ }^{83}$ M. Bona,${ }^{79}$ J. S. Bonilla, ${ }^{118}$ M. Boonekamp, ${ }^{138}$ A. Borisov, ${ }^{132}$ G. Borissov, ${ }^{75}$ J. Bortfeldt, ${ }^{32}$ D. Bortoletto, ${ }^{122}$ V. Bortolotto, ${ }^{62 a}$ D. Boscherini, ${ }^{22 a}$ M. Bosman, ${ }^{13}$ J. D. Bossio Sola, ${ }^{29}$ J. Boudreau, ${ }^{127}$

E. V. Bouhova-Thacker, ${ }^{75}$ D. Boumediene,${ }^{37}$ C. Bourdarios, ${ }^{119}$ S. K. Boutle ${ }^{56}$ A. Boveia, ${ }^{113}$ J. Boyd, ${ }^{32}$ I. R. Boyko, ${ }^{68}$ A. J. Bozson, ${ }^{80}$ J. Bracinik, ${ }^{19}$ A. Brandt, ${ }^{8}$ G. Brandt, ${ }^{177}$ O. Brandt, ${ }^{60 a}$ F. Braren, ${ }^{45}$ U. Bratzler, ${ }^{158}$ B. Brau, ${ }^{89}$ J. E. Brau, ${ }^{118}$ W. D. Breaden Madden, ${ }^{56}$ K. Brendlinger, ${ }^{45}$ A. J. Brennan, ${ }^{91}$ L. Brenner, ${ }^{45}$ R. Brenner, ${ }^{168}$ S. Bressler, ${ }^{175}$ D. L. Briglin, ${ }^{19}$ T. M. Bristow, ${ }^{49}$ D. Britton, ${ }^{56}$ D. Britzger, ${ }^{60 \mathrm{~b}}$ I. Brock, ${ }^{23}$ R. Brock,${ }^{93}$ G. Brooijmans, ${ }^{38}$ T. Brooks, ${ }^{80}$ W. K. Brooks,${ }^{34 \mathrm{~b}}$ E. Brost, ${ }^{110}$ J. H Broughton, ${ }^{19}$ P. A. Bruckman de Renstrom, ${ }^{42}$ D. Bruncko, ${ }^{146 \mathrm{~b}}$ A. Bruni, ${ }^{22 \mathrm{a}}$ G. Bruni, ${ }^{22 \mathrm{a}}$ L. S. Bruni, ${ }^{109}$ S. Bruno, ${ }^{135 a, 135 b}$ BH Brunt,${ }^{30}$ M. Bruschi, ${ }^{22 a}$ N. Bruscino, ${ }^{127}$ P. Bryant, ${ }^{33}$ L. Bryngemark,${ }^{45}$ T. Buanes, ${ }^{15}$ Q. Buat, ${ }^{32}$ P. Buchholz, ${ }^{143}$ A. G. Buckley, ${ }^{56}$ I. A. Budagov, ${ }^{68}$ F. Buehrer,${ }^{51}$ M. K. Bugge,${ }^{121}$ O. Bulekov, ${ }^{100}$ D. Bullock, ${ }^{8}$ T. J. Burch, ${ }^{110}$ S. Burdin, ${ }^{77}$ C. D. Burgard, ${ }^{109}$ A. M. Burger,${ }^{5}$ B. Burghgrave, ${ }^{110}$ K. Burka, ${ }^{42}$ S. Burke, ${ }^{133}$ I. Burmeister,${ }^{46}$ J. T. P. Burr, ${ }^{122}$ D. Büscher, ${ }^{51}$ V. Büscher, ${ }^{86}$ E. Buschmann, ${ }^{58}$ P. Bussey, ${ }^{56}$ J. M. Butler ${ }^{24}$ C. M. Buttar, ${ }^{56}$ J. M. Butterworth, ${ }^{81}$ P. Butti, ${ }^{32}$ W. Buttinger, ${ }^{32}$ A. Buzatu, ${ }^{153}$ A. R. Buzykaev, ${ }^{11, d}$ G. Cabras, ${ }^{22 a, 22 b}$ S. Cabrera Urbán, ${ }^{170}$ D. Caforio, ${ }^{130}$ H. Cai, ${ }^{169}$ V. M. M. Cairo, ${ }^{2}$ O. Cakir, ${ }^{4 a}$ N. Calace, ${ }^{52}$ P. Calafiura, ${ }^{16}$ A. Calandri, ${ }^{88}$ G. Calderini,${ }^{83}$ P. Calfayan, ${ }^{64}$ G. Callea, ${ }^{40 a, 40 b}$ L. P. Caloba, ${ }^{26 a}$ S. Calvente Lopez, ${ }^{85}$ D. Calvet, ${ }^{37}$ S. Calvet, ${ }^{37}$ T. P. Calvet, ${ }^{88}$ M. Calvetti, ${ }^{126 a, 126 b}$ R. Camacho Toro, ${ }^{33}$ S. Camarda, ${ }^{32}$ P. Camarri, ${ }^{135 a, 135 b}$ D. Cameron, ${ }^{121}$ R. Caminal Armadans, ${ }^{89}$ C. Camincher,${ }^{57}$ S. Campana, ${ }^{32}$ M. Campanelli, ${ }^{81}$ A. Camplani, ${ }^{94 a, 94 b}$ A. Campoverde, ${ }^{143}$ V. Canale, ${ }^{106 a, 106 b}$ M. Cano Bret, ${ }^{36 \mathrm{c}}$ J. Cantero, ${ }^{116}$ T. Cao, ${ }^{155}$ Y. Cao, ${ }^{169}$ M. D. M. Capeans Garrido, ${ }^{32}$ I. Caprini, ${ }^{28 \mathrm{~b}}$ M. Caprini,${ }^{28 \mathrm{~b}}$ M. Capua, ${ }^{40 \mathrm{a}, 40 \mathrm{~b}}$ R. M. Carbone,${ }^{38}$ R. Cardarelli, ${ }^{135 \mathrm{a}}$ F. Cardillo, ${ }^{51}$ I. Carli, ${ }^{131}$ T. Carli, ${ }^{32}$ G. Carlino, ${ }^{106 a}$ B. T. Carlson, ${ }^{127}$ L. Carminati, ${ }^{94 a, 94 b}$ R. M. D. Carney, ${ }^{148 a, 148 b}$ S. Caron, ${ }^{108}$ E. Carquin, ${ }^{34 \mathrm{~b}}$ S. Carrá, ${ }^{94 a, 94 b}$ G. D. Carrillo-Montoya, ${ }^{32}$ D. Casadei, ${ }^{19}$ M. P. Casado, ${ }^{13, k}$ A. F. Casha, ${ }^{161}$ M. Casolino, ${ }^{13}$ D. W. Casper, ${ }^{166}$ R. Castelijn, ${ }^{109}$ V. Castillo Gimenez, ${ }^{170}$ N. F. Castro, ${ }^{128 a}$ A. Catinaccio, ${ }^{32}$ J. R. Catmore, ${ }^{121}$ A. Cattai, ${ }^{32}$ J. Caudron, ${ }^{23}$ V. Cavaliere, ${ }^{27}$ E. Cavallaro, ${ }^{13}$ D. Cavalli, ${ }^{94 \mathrm{a}}$ M. Cavalli-Sforza ${ }^{13}$ V. Cavasinni, ${ }^{126 a, 126 \mathrm{~b}}$ E. Celebi, ${ }^{20 \mathrm{~d}}$ F. Ceradini, ${ }^{136 a, 136 b}$ L. Cerda Alberich, ${ }^{170}$ A. S. Cerqueira, ${ }^{26 b}$ A. Cerri, ${ }^{151}$ L. Cerrito, ${ }^{135 a, 135 b}$ F. Cerutti, ${ }^{16}$ A. Cervelli, ${ }^{22 a, 22 b}$ S. A. Cetin, ${ }^{20 \mathrm{~d}}$ A. Chafaq, ${ }^{137 a}$ D. Chakraborty, ${ }^{110}$ S. K. Chan, ${ }^{59}$ W. S. Chan, ${ }^{109}$ Y. L. Chan, ${ }^{62 a}$ P. Chang, ${ }^{169}$ J. D. Chapman ${ }^{30}$ D. G. Charlton, ${ }^{19}$ C. C. Chau, ${ }^{31}$ C. A. Chavez Barajas, ${ }^{151}$ S. Che, ${ }^{113}$ A. Chegwidden, ${ }^{93}$ S. Chekanov, ${ }^{6}$ S. V. Chekulaev, ${ }^{163 a}$ G. A. Chelkov, ${ }^{68,1}$ M. A. Chelstowska, ${ }^{32}$ C. Chen ${ }^{36 a}$ C. Chen,${ }^{67}$ H. Chen, ${ }^{27}$ J. Chen ${ }^{36 a}$ J. Chen, ${ }^{38}$ S. Chen,${ }^{35 b}$ S. Chen, ${ }^{124}$ X. Chen, ${ }^{35 c, m}$ Y. Chen, ${ }^{70}$ H. C. Cheng, ${ }^{92}$ H. J. Cheng, ${ }^{35 a, 35 d}$ A. Cheplakov,${ }^{68}$ E. Cheremushkina, ${ }^{132}$

R. Cherkaoui El Moursli, ${ }^{137 e}$ E. Cheu, ${ }^{7}$ K. Cheung, ${ }^{63}$ L. Chevalier, ${ }^{138}$ V. Chiarella, ${ }^{50}$ G. Chiarelli, ${ }^{126 a}$ G. Chiodini, ${ }^{76 a}$ A. S. Chisholm, ${ }^{32}$ A. Chitan, ${ }^{28 b}$ I. Chiu, ${ }^{157}$ Y. H. Chiu, ${ }^{172}$ M. V. Chizhov, ${ }^{68}$ K. Choi, ${ }^{64}$ A. R. Chomont, ${ }^{37}$ S. Chouridou, ${ }^{156}$ Y. S. Chow, ${ }^{109}$ V. Christodoulou, ${ }^{81}$ M. C. Chu, ${ }^{62 a}$ J. Chudoba, ${ }^{129}$ A. J. Chuinard, ${ }^{90}$ J. J. Chwastowski, ${ }^{42}$ L. Chytka, ${ }^{117}$ D. Cinca, ${ }^{46}$ V. Cindro, ${ }^{78}$ I. A. Cioară, ${ }^{23}$ A. Ciocio,${ }^{16}$ F. Cirotto, ${ }^{106 a, 106 b}$ Z. H. Citron, ${ }^{175}$ M. Citterio, ${ }^{94 a}$ A. Clark, ${ }^{52}$ M. R. Clark, ${ }^{38}$ P. J. Clark, ${ }^{49}$ R. N. Clarke, ${ }^{16}$ C. Clement, ${ }^{148 a, 148 b}$ Y. Coadou, ${ }^{88}$ M. Cobal, ${ }^{167 a, 167 c}$ A. Coccaro, ${ }^{53 a, 53 b}$ J. Cochran, ${ }^{67}$ L. Colasurdo, ${ }^{108}$ B. Cole,${ }^{38}$ A. P. Colijn, ${ }^{109}$ J. Collot,${ }^{57}$ P. Conde Muiño, ${ }^{128 a, 128 b}$ E. Coniavitis, ${ }^{51}$ S. H. Connell, ${ }^{147 \mathrm{~b}}$ I. A. Connelly, ${ }^{87}$ S. Constantinescu, ${ }^{28 \mathrm{~b}}$ G. Conti, ${ }^{32}$ F. Conventi, ${ }^{106 a, n}$ A. M. Cooper-Sarkar, ${ }^{122}$ F. Cormier, ${ }^{171}$ K. J. R. Cormier, ${ }^{161}$ M. Corradi, ${ }^{134 a, 134 b}$ E. E. Corrigan, ${ }^{84}$ F. Corriveau, ${ }^{90,0}$ A. Cortes-Gonzalez, ${ }^{32}$ M. J. Costa, ${ }^{170}$ D. Costanzo, ${ }^{141}$ G. Cottin,${ }^{30}$ G. Cowan, ${ }^{80}$ B. E. Cox,${ }^{87}$ K. Cranmer, ${ }^{112}$ S. J. Crawley, ${ }^{56}$ R. A. Creager, ${ }^{124}$ G. Cree, ${ }^{31}$ S. Crépé-Renaudin, ${ }^{57}$ F. Crescioli, ${ }^{83}$ M. Cristinziani, ${ }^{23}$ V. Croft, ${ }^{112}$ G. Crosetti, ${ }^{40 a, 40 b}$ A. Cueto, ${ }^{85}$ T. Cuhadar Donszelmann, ${ }^{141}$ A. R. Cukierman, ${ }^{145}$ J. Cummings, ${ }^{179}$ M. Curatolo, ${ }^{50}$ J. Cúth, ${ }^{86}$ S. Czekierda, ${ }^{42}$ P. Czodrowski, ${ }^{32}$ G. D'amen, ${ }^{22 a, 22 b}$ S. D'Auria, ${ }^{56}$ L. D'eramo, ${ }^{83}$ M. D’Onofrio, ${ }^{77}$ M. J. Da Cunha Sargedas De Sousa, ${ }^{128 a, 128 b}$ C. Da Via,${ }^{87}$ W. Dabrowski, ${ }^{41 a}$ T. Dado, ${ }^{146 a}$ S. Dahbi, ${ }^{137 \mathrm{e}}$ T. Dai, ${ }^{92}$ O. Dale,${ }^{15}$ F. Dallaire, ${ }^{97}$ C. Dallapiccola,${ }^{89}$ M. Dam, ${ }^{39}$ J. R. Dandoy, ${ }^{124}$ M. F. Daneri, ${ }^{29}$ N. P. Dang, ${ }^{176, f}$ N. S. Dann, ${ }^{87}$ M. Danninger, ${ }^{171}$ M. Dano Hoffmann, ${ }^{138}$ V. Dao, ${ }^{32}$ G. Darbo, ${ }^{53 a}$ S. Darmora, ${ }^{8}$ O. Dartsi, ${ }^{5}$ A. Dattagupta, ${ }^{118}$ T. Daubney, ${ }^{45}$ W. Davey, ${ }^{23}$ C. David, ${ }^{45}$ T. Davidek,${ }^{131}$ D. R. Davis, ${ }^{48}$ P. Davison, ${ }^{81}$ E. Dawe, ${ }^{91}$ I. Dawson, ${ }^{141}$ K. De,${ }^{8}$ R. de Asmundis, ${ }^{106 a}$ A. De Benedetti, ${ }^{115}$ S. De Castro, ${ }^{22 a, 22 b}$ S. De Cecco, ${ }^{83}$ 
N. De Groot, ${ }^{108}$ P. de Jong, ${ }^{109}$ H. De la Torre, ${ }^{93}$ F. De Lorenzi, ${ }^{67}$ A. De Maria, ${ }^{58}$ D. De Pedis, ${ }^{134 a}$ A. De Salvo, ${ }^{134 a}$ U. De Sanctis, ${ }^{135 a, 135 b}$ A. De Santo, ${ }^{151}$ K. De Vasconcelos Corga, ${ }^{88}$ J. B. De Vivie De Regie, ${ }^{119}$ C. Debenedetti, ${ }^{139}$ D. V. Dedovich, ${ }^{68}$ N. Dehghanian, ${ }^{3}$ I. Deigaard ${ }^{109}$ M. Del Gaudio, ${ }^{40,40 b}$ J. Del Peso, ${ }^{85}$ D. Delgove, ${ }^{119}$ F. Deliot, ${ }^{138}$ C. M. Delitzsch, ${ }^{7}$ A. Dell'Acqua, ${ }^{32}$ L. Dell'Asta, ${ }^{24}$ M. Della Pietra, ${ }^{106 a, 106 b}$ D. della Volpe,${ }^{52}$ M. Delmastro, ${ }^{5}$ C. Delporte,${ }^{119}$ P. A. Delsart, ${ }^{57}$ D. A. DeMarco, ${ }^{161}$ S. Demers, ${ }^{179}$ M. Demichev ${ }^{68}$ S. P. Denisov, ${ }^{132}$ D. Denysiuk, ${ }^{109}$ D. Derendarz, ${ }^{42}$ J. E. Derkaoui, ${ }^{137 d}$ F. Derue ${ }^{83}$ P. Dervan, ${ }^{77}$ K. Desch, ${ }^{23}$ C. Deterre,${ }^{45}$ K. Dette, ${ }^{161}$ M. R. Devesa, ${ }^{29}$ P. O. Deviveiros,${ }^{32}$ A. Dewhurst ${ }^{133}$ S. Dhaliwal, ${ }^{25}$ F. A. Di Bello, ${ }^{52}$ A. Di Ciaccio, ${ }^{135 a, 135 b}$ L. Di Ciaccio, ${ }^{5}$ W. K. Di Clemente, ${ }^{124}$ C. Di Donato, ${ }^{106 a, 106 b}$ A. Di Girolamo, ${ }^{32}$ B. Di Micco, ${ }^{136 a, 136 b}$ R. Di Nardo, ${ }^{32}$ K. F. Di Petrillo, ${ }^{59}$ A. Di Simone, ${ }^{51}$ R. Di Sipio, ${ }^{161}$ D. Di Valentino, ${ }^{31}$ C. Diaconu, ${ }^{88}$ M. Diamond, ${ }^{161}$ F. A. Dias, ${ }^{39}$ M. A. Diaz, ${ }^{34 a}$ J. Dickinson, ${ }^{16}$ E. B. Diehl, ${ }^{92}$ J. Dietrich, ${ }^{17}$ S. Díez Cornell, ${ }^{45}$ A. Dimitrievska, ${ }^{16}$ J. Dingfelder, ${ }^{23}$ P. Dita, ${ }^{28 b}$ S. Dita, ${ }^{28 b}$ F. Dittus, ${ }^{32}$ F. Djama, ${ }^{88}$ T. Djobava, ${ }^{54 \mathrm{~b}}$ J. I. Djuvsland, ${ }^{60 \mathrm{a}}$ M. A. B. do Vale, ${ }^{26 \mathrm{c}}$ M. Dobre, ${ }^{28 \mathrm{~b}}$ D. Dodsworth, ${ }^{25}$ C. Doglioni,${ }^{84}$ J. Dolejsi, ${ }^{131}$ Z. Dolezal ${ }^{131}$ M. Donadelli, ${ }^{26 d}$ J. Donini, ${ }^{37}$ J. Dopke, ${ }^{133}$ A. Doria, ${ }^{106 a}$ M. T. Dova ${ }^{74}$ A. T. Doyle, ${ }^{56}$ E. Drechsler,${ }^{58}$ E. Dreyer,${ }^{144}$ M. Dris,${ }^{10}$ Y. Du, ${ }^{36 b}$ J. Duarte-Campderros, ${ }^{155}$ F. Dubinin, ${ }^{98}$ A. Dubreuil, ${ }^{52}$ E. Duchovni, ${ }^{175}$ G. Duckeck, ${ }^{102}$ A. Ducourthial ${ }^{83}$ O. A. Ducu, ${ }^{97, p}$ D. Duda ${ }^{109}$ A. Dudarev, ${ }^{32}$ A. Chr. Dudder, ${ }^{86}$ E. M. Duffield, ${ }^{16}$ L. Duflot, ${ }^{119}$ M. Dührssen, ${ }^{32}$ C. Dulsen, ${ }^{177}$ M. Dumancic, ${ }^{175}$ A. E. Dumitriu, ${ }^{28 b, q}$ A. K. Duncan, ${ }^{56}$ M. Dunford, ${ }^{60 a}$ A. Duperrin,${ }^{88}$ H. Duran Yildiz, ${ }^{4 \mathrm{a}}$ M. Düren, ${ }^{55}$ A. Durglishvili, ${ }^{54 \mathrm{~b}}$ D. Duschinger, ${ }^{47}$ B. Dutta, ${ }^{45}$ D. Duvnjak, ${ }^{1}$ M. Dyndal, ${ }^{45}$ B. S. Dziedzic,${ }^{42}$ C. Eckardt ${ }^{45}$ K. M. Ecker ${ }^{103}$ R. C. Edgar, ${ }^{92}$ T. Eifert, ${ }^{32}$ G. Eigen, ${ }^{15}$ K. Einsweiler,${ }^{16}$ T. Ekelof, ${ }^{168}$ M. El Kacimi, ${ }^{137 \mathrm{c}}$ R. El Kosseifi, ${ }^{88}$ V. Ellajosyula, ${ }^{88}$ M. Ellert, ${ }^{168}$ F. Ellinghaus, ${ }^{177}$ A. A. Elliot, ${ }^{172}$ N. Ellis, ${ }^{32}$ J. Elmsheuser ${ }^{27}$ M. Elsing ${ }^{32}$ D. Emeliyanov, ${ }^{133}$ Y. Enari, ${ }^{157}$ J. S. Ennis, ${ }^{173}$ M. B. Epland, ${ }^{48}$ J. Erdmann, ${ }^{46}$ A. Ereditato, ${ }^{18}$ S. Errede,${ }^{169}$ M. Escalier, ${ }^{119}$ C. Escobar,${ }^{170}$ B. Esposito,${ }^{50}$ O. Estrada Pastor, ${ }^{170}$ A. I. Etienvre, ${ }^{138}$ E. Etzion, ${ }^{155}$ H. Evans, ${ }^{64}$ A. Ezhilov, ${ }^{125}$ M. Ezzi, ${ }^{137 e}$ F. Fabbri, ${ }^{22 a, 22 b}$ L. Fabbri, ${ }^{22 a, 22 b}$ V. Fabiani, ${ }^{108}$ G. Facini ${ }^{81}$ R. M. Fakhrutdinov, ${ }^{132}$ S. Falciano, ${ }^{134 a}$ J. Faltova, ${ }^{131}$ Y. Fang, ${ }^{35 a}$ M. Fanti, ${ }^{94 a, 94 b}$ A. Farbin, ${ }^{8}$ A. Farilla, ${ }^{136 a}$ E. M. Farina, ${ }^{123 a, 123 b}$ T. Farooque, ${ }^{93}$ S. Farrell,${ }^{16}$ S. M. Farrington, ${ }^{173}$ P. Farthouat ${ }^{32}$

F. Fassi, ${ }^{137 \mathrm{e}}$ P. Fassnacht, ${ }^{32}$ D. Fassouliotis, ${ }^{9}$ M. Faucci Giannelli, ${ }^{49}$ A. Favareto, ${ }^{53 a, 53 b}$ W. J. Fawcett, ${ }^{52}$ L. Fayard,${ }^{119}$ O. L. Fedin, ${ }^{125, \mathrm{r}}$ W. Fedorko, ${ }^{171}$ M. Feickert, ${ }^{43}$ S. Feigl,${ }^{121}$ L. Feligioni, ${ }^{88}$ C. Feng, ${ }^{36 b}$ E. J. Feng, ${ }^{32}$ M. Feng, ${ }^{48}$ M. J. Fenton, ${ }^{56}$ A. B. Fenyuk, ${ }^{132}$ L. Feremenga, ${ }^{8}$ P. Fernandez Martinez, ${ }^{170}$ J. Ferrando, ${ }^{45}$ A. Ferrari, ${ }^{168}$ P. Ferrari, ${ }^{109}$ R. Ferrari, ${ }^{123 a}$

D. E. Ferreira de Lima, ${ }^{60 \mathrm{~b}}$ A. Ferrer, ${ }^{170}$ D. Ferrere,${ }^{52}$ C. Ferretti, ${ }^{92}$ F. Fiedler, ${ }^{86}$ A. Filipčič ${ }^{78}$ F. Filthaut, ${ }^{108}$

M. Fincke-Keeler, ${ }^{172}$ K. D. Finelli, ${ }^{24}$ M. C. N. Fiolhais, ${ }^{128 a, 128 c, s}$ L. Fiorini, ${ }^{170}$ C. Fischer, ${ }^{13}$ J. Fischer, ${ }^{177}$ W. C. Fisher, ${ }^{93}$

N. Flaschel, ${ }^{45}$ I. Fleck, ${ }^{143}$ P. Fleischmann, ${ }^{92}$ R. R. M. Fletcher, ${ }^{124}$ T. Flick, ${ }^{177}$ B. M. Flierl,,${ }^{102}$ L. M. Flores, ${ }^{124}$

L. R. Flores Castillo, ${ }^{62 a}$ N. Fomin, ${ }^{15}$ G. T. Forcolin, ${ }^{87}$ A. Formica, ${ }^{138}$ F. A. Förster, ${ }^{13}$ A. Forti, ${ }^{87}$ A. G. Foster, ${ }^{19}$

D. Fournier, ${ }^{119}$ H. Fox, ${ }^{75}$ S. Fracchia, ${ }^{141}$ P. Francavilla, ${ }^{126 a, 126 b}$ M. Franchini, ${ }^{22 a, 22 b}$ S. Franchino, ${ }^{60 a}$ D. Francis,${ }^{32}$ L. Franconi, ${ }^{121}$ M. Franklin, ${ }^{59}$ M. Frate, ${ }^{166}$ M. Fraternali, ${ }^{123 a, 123 b}$ D. Freeborn, ${ }^{81}$ S. M. Fressard-Batraneanu, ${ }^{32}$ B. Freund, ${ }^{97}$ W. S. Freund, ${ }^{26 a}$ D. Froidevaux ${ }^{32}$ J. A. Frost, ${ }^{122}$ C. Fukunaga ${ }^{158}$ T. Fusayasu, ${ }^{104}$ J. Fuster, ${ }^{170}$ O. Gabizon, ${ }^{154}$ A. Gabrielli, ${ }^{22 a, 22 b}$ A. Gabrielli, ${ }^{16}$ G. P. Gach, ${ }^{41 a}$ S. Gadatsch, ${ }^{52}$ S. Gadomski, ${ }^{80}$ P. Gadow, ${ }^{103}$ G. Gagliardi, ${ }^{53,53 b}$ L. G. Gagnon, ${ }^{97}$ C. Galea, ${ }^{108}$ B. Galhardo, ${ }^{128 a, 128 c}$ E. J. Gallas, ${ }^{122}$ B. J. Gallop, ${ }^{133}$ P. Gallus, ${ }^{130}$ G. Galster, ${ }^{39}$ R. Gamboa Goni, ${ }^{79}$ K. K. Gan, ${ }^{113}$ S. Ganguly, ${ }^{175}$ Y. Gao, ${ }^{77}$ Y. S. Gao, ${ }^{145, h}$ F. M. Garay Walls, ${ }^{34 a}$ C. García, ${ }^{170}$ J. E. García Navarro, ${ }^{170}$ J. A. García Pascual, ${ }^{35 a}$ M. Garcia-Sciveres, ${ }^{16}$ R. W. Gardner, ${ }^{33}$ N. Garelli, ${ }^{145}$ V. Garonne, ${ }^{121}$

K. Gasnikova, ${ }^{45}$ A. Gaudiello, ${ }^{53 a, 53 b}$ G. Gaudio, ${ }^{123 a}$ I. L. Gavrilenko, ${ }^{98}$ C. Gay, ${ }^{171}$ G. Gaycken, ${ }^{23}$ E. N. Gazis, ${ }^{10}$ C. N. P. Gee, ${ }^{133}$ J. Geisen, ${ }^{58}$ M. Geisen, ${ }^{86}$ M. P. Geisler, ${ }^{60 a}$ K. Gellerstedt, ${ }^{148 a, 148 b}$ C. Gemme, ${ }^{53 \mathrm{a}}$ M. H. Genest, ${ }^{57}$ C. Geng, ${ }^{92}$ S. Gentile, ${ }^{134 a, 134 b}$ C. Gentsos, ${ }^{156}$ S. George, ${ }^{80}$ D. Gerbaudo, ${ }^{13}$ G. Geßner, ${ }^{46}$ S. Ghasemi, ${ }^{143}$ M. Ghneimat, ${ }^{23}$ B. Giacobbe, ${ }^{22 a}$ S. Giagu, ${ }_{134 a, 134 b}$ N. Giangiacomi, ${ }^{22 a, 22 b}$ P. Giannetti, ${ }^{126 a}$ S. M. Gibson, ${ }^{80}$ M. Gignac, ${ }^{139}$ M. Gilchriese, ${ }^{16}$ D. Gillberg, ${ }^{31}$ G. Gilles, ${ }^{177}$ D. M. Gingrich, ${ }^{3, \mathrm{e}}$ M. P. Giordani, ${ }^{167 \mathrm{a}, 167 \mathrm{c}}$ F. M. Giorgi, ${ }^{22 \mathrm{a}}$ P. F. Giraud,${ }^{138}$ P. Giromini,${ }^{59}$ G. Giugliarelli, ${ }^{167 \mathrm{a}, 167 \mathrm{c}}$ D. Giugni, ${ }^{94 a}$ F. Giuli, ${ }^{122}$ M. Giulini, ${ }^{60 b}$ S. Gkaitatzis, ${ }^{156}$ I. Gkialas, ${ }^{9, t}$ E. L. Gkougkousis, ${ }^{13}$ P. Gkountoumis, ${ }^{10}$ L. K. Gladilin, ${ }^{101}$ C. Glasman, ${ }^{85}$ J. Glatzer, ${ }^{13}$ P. C. F. Glaysher, ${ }^{45}$ A. Glazov, ${ }^{45}$ M. Goblirsch-Kolb, ${ }^{25}$ J. Godlewski, ${ }^{42}$ S. Goldfarb, ${ }^{91}$ T. Golling, ${ }^{52}$ D. Golubkov, ${ }^{132}$ A. Gomes, ${ }^{128 a, 128 b, 128 d}$ R. Gonçalo, ${ }^{128 a}$ R. Goncalves Gama, ${ }^{26 b}$ G. Gonella, ${ }^{51}$ L. Gonella, ${ }^{19}$ A. Gongadze, ${ }^{68}$ F. Gonnella, ${ }^{19}$ J. L. Gonski, ${ }^{59}$ S. González de la Hoz,${ }^{170}$ S. Gonzalez-Sevilla, ${ }^{52}$ L. Goossens, ${ }^{32}$ P. A. Gorbounov, ${ }^{99}$ H. A. Gordon,${ }^{27}$ B. Gorini ${ }^{32}$ E. Gorini, ${ }^{76 a, 76 b}$ A. Gorišek, ${ }^{78}$ A. T. Goshaw, ${ }^{48}$ C. Gössling, ${ }^{46}$ M. I. Gostkin ${ }^{68}$ C. A. Gottardo, ${ }^{23}$ C. R. Goudet, ${ }^{119}$ D. Goujdami, ${ }^{137 \mathrm{c}}$ A. G. Goussiou, ${ }^{140}$ N. Govender, ${ }^{147 b, u}$ C. Goy, ${ }^{5}$ E. Gozani, ${ }^{154}$ I. Grabowska-Bold, ${ }^{41 a}$ P. O. J. Gradin, ${ }^{168}$ E. C. Graham, ${ }^{77}$ J. Gramling, ${ }^{166}$ E. Gramstad, ${ }^{121}$ S. Grancagnolo, ${ }^{17}$ V. Gratchev, ${ }^{125}$ P. M. Gravila, ${ }^{28 f}$ C. Gray, ${ }^{56}$ H. M. Gray, ${ }^{16}$ Z. D. Greenwood, ${ }^{82, v}$ C. Grefe, ${ }^{23}$ K. Gregersen, ${ }^{81}$ I. M. Gregor, ${ }^{45}$ 
P. Grenier, ${ }^{145}$ K. Grevtsov, ${ }^{45}$ J. Griffiths, ${ }^{8}$ A. A. Grillo, ${ }^{139}$ K. Grimm, ${ }^{145}$ S. Grinstein, ${ }^{13, w}$ Ph. Gris, ${ }^{37}$ J.-F. Grivaz, ${ }^{119}$ S. Groh, ${ }^{86}$ E. Gross,${ }^{175}$ J. Grosse-Knetter, ${ }^{58}$ G. C. Grossi, ${ }^{82}$ Z. J. Grout, ${ }^{81}$ A. Grummer, ${ }^{107}$ L. Guan, ${ }^{92}$ W. Guan, ${ }^{176}$ J. Guenther, ${ }^{32}$ A. Guerguichon, ${ }^{119}$ F. Guescini, ${ }^{163 a}$ D. Guest, ${ }^{166}$ O. Gueta, ${ }^{155}$ R. Gugel,${ }^{51}$ B. Gui, ${ }^{113}$ T. Guillemin, ${ }^{5}$ S. Guindon, ${ }^{32}$ U. Gul,${ }^{56}$ C. Gumpert, ${ }^{32}$ J. Guo, ${ }^{36 \mathrm{c}}$ W. Guo, ${ }^{92}$ Y. Guo,${ }^{36 a, x}$ R. Gupta, ${ }^{43}$ S. Gurbuz, ${ }^{20 a}$ G. Gustavino, ${ }^{115}$ B. J. Gutelman, ${ }^{154}$ P. Gutierrez, ${ }^{115}$ N. G. Gutierrez Ortiz, ${ }^{81}$ C. Gutschow, ${ }^{81}$ C. Guyot, ${ }^{138}$ M. P. Guzik, ${ }^{41 a}$ C. Gwenlan, ${ }^{122}$ C. B. Gwilliam, ${ }^{77}$ A. Haas, ${ }^{112}$ C. Haber, ${ }^{16}$ H. K. Hadavand, ${ }^{8}$ N. Haddad, ${ }^{137 e}$ A. Hadef ${ }^{88}$ S. Hageböck, ${ }^{23}$ M. Hagihara, ${ }^{164}$ H. Hakobyan, ${ }^{180, a}$ M. Haleem, ${ }^{178}$ J. Haley, ${ }^{116}$ G. Halladjian, ${ }^{93}$ G. D. Hallewell ${ }^{88}$ K. Hamacher, ${ }^{177}$ P. Hamal, ${ }^{117}$ K. Hamano, ${ }^{172}$ A. Hamilton, ${ }^{147 a}$ G. N. Hamity, ${ }^{141}$ K. Han, ${ }^{36 a, y}$ L. Han,${ }^{36 a}$ S. Han, ${ }^{35 a, 35 d}$ K. Hanagaki, ${ }^{69, z}$ M. Hance, ${ }^{139}$ D. M. Handl, ${ }^{102}$ B. Haney, ${ }^{124}$ R. Hankache ${ }^{83}$ P. Hanke, ${ }^{60 a}$ E. Hansen, ${ }^{84}$ J. B. Hansen, ${ }^{39}$ J. D. Hansen, ${ }^{39}$ M. C. Hansen, ${ }^{23}$ P. H. Hansen, ${ }^{39}$ K. Hara, ${ }^{164}$ A. S. Hard,${ }^{176}$ T. Harenberg, ${ }^{177}$ S. Harkusha, ${ }^{95}$ P. F. Harrison, ${ }^{173}$ N. M. Hartmann, ${ }^{102}$ Y. Hasegawa, ${ }^{142}$ A. Hasib, ${ }^{49}$ S. Hassani, ${ }^{138}$ S. Haug, ${ }^{18}$ R. Hauser, ${ }^{93}$ L. Hauswald, ${ }^{47}$ L. B. Havener, ${ }^{38}$ M. Havranek, ${ }^{130}$ C. M. Hawkes, ${ }^{19}$ R. J. Hawkings, ${ }^{32}$ D. Hayden,${ }^{93}$ C. P. Hays,${ }^{122}$ J. M. Hays,${ }^{79}$ H. S. Hayward,${ }^{77}$ S. J. Haywood, ${ }^{133}$ T. Heck,${ }^{86}$ V. Hedberg, ${ }^{84}$ L. Heelan, ${ }^{8}$ S. Heer, ${ }^{23}$ K. K. Heidegger, ${ }^{51}$ S. Heim, ${ }^{45}$ T. Heim,${ }^{16}$ B. Heinemann, ${ }^{45, \text { aa }}$ J. J. Heinrich, ${ }^{102}$

L. Heinrich, ${ }^{112}$ C. Heinz, ${ }^{55}$ J. Hejbal, ${ }^{129}$ L. Helary, ${ }^{32}$ A. Held, ${ }^{171}$ S. Hellesund, ${ }^{121}$ S. Hellman, ${ }^{148 a, 148 b}$ C. Helsens, ${ }^{32}$ R. C. W. Henderson, ${ }^{75}$ Y. Heng, ${ }^{176}$ S. Henkelmann, ${ }^{171}$ A. M. Henriques Correia,${ }^{32}$ G. H. Herbert,${ }^{17}$ H. Herde, ${ }^{25}$ V. Herget, ${ }^{178}$ Y. Hernández Jiménez, ${ }^{147 \mathrm{c}}$ H. Herr ${ }^{86}$ G. Herten ${ }^{51}$ R. Hertenberger, ${ }^{102}$ L. Hervas, ${ }^{32}$ T. C. Herwig, ${ }^{124}$ G. G. Hesketh, ${ }^{81}$ N. P. Hessey, ${ }^{163 a}$ J. W. Hetherly, ${ }^{43}$ S. Higashino, ${ }^{69}$ E. Higón-Rodriguez, ${ }^{170}$ K. Hildebrand,${ }^{33}$ E. Hill, ${ }^{172}$ J. C. Hill ${ }^{30}$ K. H. Hiller, ${ }^{45}$ S. J. Hillier, ${ }^{19}$ M. Hils ${ }^{47}$ I. Hinchliffe, ${ }^{16}$ M. Hirose,${ }^{51}$ D. Hirschbuehl, ${ }^{177}$ B. Hiti, ${ }^{78}$ O. Hladik, ${ }^{129}$ D. R. Hlaluku, ${ }^{147 \mathrm{c}}$ X. Hoad, ${ }^{49}$ J. Hobbs,${ }^{150}$ N. Hod, ${ }^{163 a}$ M. C. Hodgkinson, ${ }^{141}$ A. Hoecker, ${ }^{32}$ M. R. Hoeferkamp, ${ }^{107}$ F. Hoenig, ${ }^{102}$ D. Hohn, ${ }^{23}$ D. Hohov, ${ }^{119}$ T. R. Holmes, ${ }^{33}$ M. Holzbock, ${ }^{102}$ M. Homann, ${ }^{46}$ S. Honda, ${ }^{164}$ T. Honda, ${ }^{69}$ T. M. Hong, ${ }^{127}$ B. H. Hooberman, ${ }^{169}$ W. H. Hopkins, ${ }^{118}$ Y. Horii, ${ }^{105}$ A. J. Horton, ${ }^{144}$ L. A. Horyn, ${ }^{33}$ J-Y. Hostachy, ${ }^{57}$ A. Hostiuc ${ }^{140}$ S. Hou, ${ }^{153}$ A. Hoummada, ${ }^{137 a}$ J. Howarth, ${ }^{87}$ J. Hoya,${ }^{74}$ M. Hrabovsky, ${ }^{17}$ J. Hrdinka, ${ }^{32}$ I. Hristova, ${ }^{17}$ J. Hrivnac, ${ }^{119}$ T. Hryn'ova, ${ }^{5}$ A. Hrynevich, ${ }^{96}$ P. J. Hsu, ${ }^{63}$ S.-C. Hsu, ${ }^{140}$ Q. Hu,${ }^{27}$ S. Hu, ${ }^{36 c}$ Y. Huang, ${ }^{35 a}$ Z. Hubacek, ${ }^{130}$ F. Hubaut ${ }^{88}$ F. Huegging, ${ }^{23}$ T. B. Huffman, ${ }^{122}$ E. W. Hughes,${ }^{38}$ M. Huhtinen, ${ }^{32}$ R. F. H. Hunter, ${ }^{31}$ P. Huo, ${ }^{150}$ A. M. Hupe, ${ }^{31}$ N. Huseynov, ${ }^{68, c}$ J. Huston, ${ }^{93}$ J. Huth, ${ }^{59}$ R. Hyneman, ${ }^{92}$ G. Iacobucci, ${ }^{52}$ G. Iakovidis, ${ }^{27}$ I. Ibragimov, ${ }^{143}$

L. Iconomidou-Fayard, ${ }^{119}$ Z. Idrissi, ${ }^{137 e}$ P. Iengo, ${ }^{32}$ O. Igonkina, ${ }^{109, b b}$ R. Iguchi, ${ }^{157}$ T. Iizawa, ${ }^{174}$ Y. Ikegami, ${ }^{69}$ M. Ikeno, ${ }^{69}$ D. Iliadis, ${ }^{156} \mathrm{~N}$. Ilic, ${ }^{145} \mathrm{~F}$. Iltzsche,${ }^{47} \mathrm{G}$. Introzzi, ${ }^{123 a, 123 b} \mathrm{M}$. Iodice, ${ }^{136 \mathrm{a}} \mathrm{K}$. Iordanidou, ${ }^{38}$ V. Ippolito, ${ }^{134 a, 134 b} \mathrm{M}$. F. Isacson, ${ }^{168}$ N. Ishijima, ${ }^{120} \mathrm{M}$. Ishino, ${ }^{157} \mathrm{M}$. Ishitsuka, ${ }^{159} \mathrm{C}$. Issever, ${ }^{122} \mathrm{~S}$. Istin, ${ }^{20 \mathrm{a}} \mathrm{F}$. Ito, ${ }^{164} \mathrm{~J}$. M. Iturbe Ponce, ${ }^{62 \mathrm{a}} \mathrm{R}$. Iuppa, ${ }^{162 a, 162 \mathrm{~b}}$ H. Iwasaki, ${ }^{69}$ J. M. Izen, ${ }^{44}$ V. Izzo, ${ }^{106 a}$ S. Jabbar, ${ }^{3}$ P. Jacka, ${ }^{129}$ P. Jackson, ${ }^{1}$ R. M. Jacobs,${ }^{23}$ V. Jain, ${ }^{2}$ G. Jakel, ${ }^{177}$ K. B. Jakobi, ${ }^{86}$ K. Jakobs, ${ }^{51}$ S. Jakobsen, ${ }^{65}$ T. Jakoubek, ${ }^{129}$ D. O. Jamin, ${ }^{116}$ D. K. Jana, ${ }^{82}$ R. Jansky, ${ }^{52}$ J. Janssen, ${ }^{23}$ M. Janus, ${ }^{58}$ P. A. Janus, ${ }^{41 a}$ G. Jarlskog, ${ }^{84}$ N. Javadov, ${ }^{68, c}$ T. Javưrek, ${ }^{51}$ M. Javurkova, ${ }^{51}$ F. Jeanneau, ${ }^{138}$ L. Jeanty, ${ }^{16}$ J. Jejelava, ${ }^{54 a, c c}$ A. Jelinskas, ${ }^{173}$ P. Jenni, ${ }^{51, d d}$ C. Jeske, ${ }^{173}$ S. Jézéquel, ${ }^{5} \mathrm{H}$. Ji, ${ }^{176}$ J. Jia, ${ }^{150}$ H. Jiang,,${ }^{67}$ Y. Jiang, ${ }^{36 a}$ Z. Jiang, ${ }^{145}$ S. Jiggins, ${ }^{81}$ J. Jimenez Pena, ${ }^{170}$ S. Jin, ${ }^{35 b}$ A. Jinaru, ${ }^{28 b}$ O. Jinnouchi, ${ }^{159}$ H. Jivan, ${ }^{147 c}$ P. Johansson, ${ }^{141}$ K. A. Johns, ${ }^{7}$ C. A. Johnson, ${ }^{64}$ W. J. Johnson, ${ }^{140}$ K. Jon-And, ${ }^{148 a, 148 b}$ R. W. L. Jones, ${ }^{75}$ S. D. Jones, ${ }^{151}$ S. Jones, ${ }^{7}$ T. J. Jones, ${ }^{77}$ J. Jongmanns ${ }^{60 \mathrm{a}}$ P. M. Jorge, ${ }^{128 a, 128 b}$ J. Jovicevic, ${ }^{163 \mathrm{a}}$ X. Ju, ${ }^{176}$ J. J. Junggeburth, ${ }^{103}$ A. Juste Rozas, ${ }^{13, w}$ A. Kaczmarska, ${ }^{42}$ M. Kado, ${ }^{119}$ H. Kagan, ${ }^{113}$ M. Kagan, ${ }^{145}$ S. J. Kahn,${ }^{88}$ T. Kaji, ${ }^{174}$ E. Kajomovitz, ${ }^{154}$ C. W. Kalderon, ${ }^{84}$ A. Kaluza, ${ }^{86}$ S. Kama, ${ }^{43}$ A. Kamenshchikov, ${ }^{132}$ L. Kanjir, ${ }^{78}$ Y. Kano, ${ }^{157}$ V. A. Kantserov, ${ }^{100}$ J. Kanzaki, ${ }^{69}$ B. Kaplan, ${ }^{112}$ L. S. Kaplan, ${ }^{176}$ D. Kar, ${ }^{147 \mathrm{c}}$ K. Karakostas, ${ }^{10}$ N. Karastathis,${ }^{10}$ M. J. Kareem, ${ }^{163 b}$ E. Karentzos, ${ }^{10}$ S. N. Karpov ${ }^{68}$ Z. M. Karpova, ${ }^{68}$ V. Kartvelishvili, ${ }^{75}$ A. N. Karyukhin, ${ }^{132}$ K. Kasahara, ${ }^{164}$ L. Kashif,${ }^{176}$ R. D. Kass, ${ }^{113}$ A. Kastanas, ${ }^{149}$ Y. Kataoka, ${ }^{157}$ C. Kato, ${ }^{157}$ A. Katre, ${ }^{52}$ J. Katzy, ${ }^{45}$ K. Kawade ${ }^{70}$ K. Kawagoe, ${ }^{73}$ T. Kawamoto, ${ }^{157}$ G. Kawamura, ${ }^{58}$ E. F. Kay ${ }^{77}$ V. F. Kazanin, ${ }^{111, \mathrm{~d}}$ R. Keeler, ${ }^{172}$ R. Kehoe, ${ }^{43}$ J. S. Keller, ${ }^{31}$ E. Kellermann, ${ }^{84}$ J. J. Kempster, ${ }^{19}$ J Kendrick, ${ }^{19}$ H. Keoshkerian, ${ }^{161}$ O. Kepka, ${ }^{129}$ B. P. Kerševan, ${ }^{78}$ S. Kersten, ${ }^{177}$ R. A. Keyes,${ }^{90}$ M. Khader, ${ }^{169}$ F. Khalil-zada, ${ }^{12}$ A. Khanov, ${ }^{116}$ A. G. Kharlamov, ${ }^{111, d}$ T. Kharlamova, ${ }^{111, d}$ A. Khodinov ${ }^{160}$ T. J. Khoo, ${ }^{52}$ V. Khovanskiy, ${ }^{99, a}$ E. Khramov, ${ }^{68}$

J. Khubua, ${ }^{54 b, e e}$ S. Kido, ${ }^{70}$ M. Kiehn, ${ }^{52}$ C. R. Kilby,${ }^{80}$ H. Y. Kim, ${ }^{8}$ S. H. Kim, ${ }^{164}$ Y. K. Kim, ${ }^{33}$ N. Kimura, ${ }^{167 a, 167 c}$ O. M. Kind, ${ }^{17}$ B. T. King, ${ }^{77}$ D. Kirchmeier, ${ }^{47}$ J. Kirk, ${ }^{133}$ A. E. Kiryunin, ${ }^{103}$ T. Kishimoto ${ }^{157}$ D. Kisielewska, ${ }^{41 a}$ V. Kitali, ${ }^{45}$ O. Kivernyk, ${ }^{5}$ E. Kladiva, ${ }^{146 \mathrm{~b}}$ T. Klapdor-Kleingrothaus, ${ }^{51}$ M. H. Klein, ${ }^{92}$ M. Klein,${ }^{77}$ U. Klein, ${ }^{77}$ K. Kleinknecht, ${ }^{86}$ P. Klimek, ${ }^{110}$ A. Klimentov, ${ }^{27}$ R. Klingenberg, ${ }^{46, a}$ T. Klingl, ${ }^{23}$ T. Klioutchnikova, ${ }^{32}$ F. F. Klitzner, ${ }^{102}$ E.-E. Kluge, ${ }^{60 a}$ P. Kluit, ${ }^{109}$ S. Kluth ${ }^{103}$ E. Kneringer, ${ }^{65}$ E. B. F. G. Knoops, ${ }^{88}$ A. Knue,${ }^{51}$ A. Kobayashi, ${ }^{157}$ D. Kobayashi, ${ }^{73}$ T. Kobayashi, ${ }^{157}$ M. Kobel, ${ }^{47}$ M. Kocian, ${ }^{145}$ P. Kodys, ${ }^{131}$ T. Koffas, ${ }^{31}$ E. Koffeman, ${ }^{109}$ N. M. Köhler, ${ }^{103}$ T. Koi, ${ }^{145}$ M. Kolb,${ }^{60 b}$ I. Koletsou, ${ }^{5}$ 
T. Kondo, ${ }^{69}$ N. Kondrashova, ${ }^{36 c}$ K. Köneke, ${ }^{51}$ A. C. König, ${ }^{108}$ T. Kono, ${ }^{69, f f}$ R. Konoplich, ${ }^{12, g g}$ N. Konstantinidis, ${ }^{81}$ B. Konya,${ }^{84}$ R. Kopeliansky, ${ }^{64}$ S. Koperny, ${ }^{41 a}$ K. Korcyl, ${ }^{42}$ K. Kordas, ${ }^{156}$ A. Korn, ${ }^{81}$ I. Korolkov, ${ }^{13}$ E. V. Korolkova, ${ }^{141}$ O. Kortner, ${ }^{103}$ S. Kortner, ${ }^{103}$ T. Kosek, ${ }^{131}$ V. V. Kostyukhin, ${ }^{23}$ A. Kotwal, ${ }^{48}$ A. Koulouris, ${ }^{10}$

A. Kourkoumeli-Charalampidi, ${ }^{123 a, 123 b}$ C. Kourkoumelis, ${ }^{9}$ E. Kourlitis,${ }^{141}$ V. Kouskoura, ${ }^{27}$ A. B. Kowalewska, ${ }^{42}$ R. Kowalewski, ${ }^{172}$ T. Z. Kowalski, ${ }^{41 a}$ C. Kozakai, ${ }^{157}$ W. Kozanecki, ${ }^{138}$ A. S. Kozhin, ${ }^{132}$ V. A. Kramarenko, ${ }^{101}$ G. Kramberger, ${ }^{78}$ D. Krasnopevtsev, ${ }^{100}$ M. W. Krasny, ${ }^{83}$ A. Krasznahorkay, ${ }^{32}$ D. Krauss, ${ }^{103}$ J. A. Kremer, ${ }^{41 a}$ J. Kretzschmar, ${ }^{77}$ K. Kreutzfeldt, ${ }^{55}$ P. Krieger, ${ }^{161}$ K. Krizka, ${ }^{16}$ K. Kroeninger, ${ }^{46}$ H. Kroha, ${ }^{103}$ J. Kroll, ${ }^{129}$ J. Kroll, ${ }^{124}$ J. Kroseberg, ${ }^{23}$ J. Krstic, ${ }^{14}$ U. Kruchonak, ${ }^{68}$ H. Krüger, ${ }^{23}$ N. Krumnack,${ }^{67}$ M. C. Kruse, ${ }^{48}$ T. Kubota, ${ }^{91}$ S. Kuday, ${ }^{4 b}$ J. T. Kuechler, ${ }^{177}$ S. Kuehn, ${ }^{32}$ A. Kugel,,${ }^{60 a}$ F. Kuger, ${ }^{178}$ T. Kuhl,${ }^{45}$ V. Kukhtin, ${ }^{68}$ R. Kukla, ${ }^{88}$ Y. Kulchitsky, ${ }^{95}$ S. Kuleshov, ${ }^{34 b}$ Y. P. Kulinich, ${ }^{169}$ M. Kuna, ${ }^{57}$ T. Kunigo,${ }^{71}$ A. Kupco, ${ }^{129}$ T. Kupfer, ${ }^{46}$ O. Kuprash, ${ }^{155}$ H. Kurashige, ${ }^{70}$ L. L. Kurchaninov, ${ }^{163 a}$ Y. A. Kurochkin, ${ }^{95}$ M. G. Kurth, ${ }^{35 a, 35 d}$ E. S. Kuwertz, ${ }^{172}$ M. Kuze, ${ }^{159}$ J. Kvita, ${ }^{117}$ T. Kwan, ${ }^{172}$ A. La Rosa, ${ }^{103}$ J. L. La Rosa Navarro, ${ }^{26 \mathrm{~d}}$ L. La Rotonda ${ }^{40 \mathrm{a}, 40 \mathrm{~b}}$ F. La Ruffa ${ }^{40 \mathrm{a}, 40 \mathrm{~b}}$ C. Lacasta,${ }^{170}$ F. Lacava, ${ }^{134 a, 134 \mathrm{~b}}$ J. Lacey, ${ }^{45}$ D. P. J. Lack, ${ }^{87}$ H. Lacker, ${ }^{17}$ D. Lacour, ${ }^{83}$ E. Ladygin, ${ }^{68}$ R. Lafaye,${ }^{5}$ B. Laforge,${ }^{83}$ S. Lai, ${ }^{58}$ S. Lammers,${ }^{64}$ W. Lampl, ${ }^{7}$ E. Lançon, ${ }^{27}$ U. Landgraf, ${ }^{51}$ M. P. J. Landon, ${ }^{79}$ M. C. Lanfermann, ${ }^{52}$ V. S. Lang, ${ }^{45}$ J. C. Lange, ${ }^{13}$ R. J. Langenberg, ${ }^{32}$ A. J. Lankford, ${ }^{166}$ F. Lanni, ${ }^{27}$ K. Lantzsch, ${ }^{23}$ A. Lanza, ${ }^{123 a}$ A. Lapertosa, ${ }^{53 a, 53 b}$ S. Laplace, ${ }^{83}$ J. F. Laporte, ${ }^{138}$ T. Lari, ${ }^{94 a}$

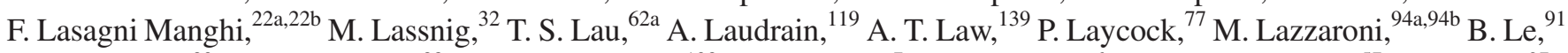
O. Le Dortz, ${ }^{83}$ E. Le Guirriec, ${ }^{88}$ E. P. Le Quilleuc, ${ }^{138}$ M. LeBlanc, ${ }^{7}$ T. LeCompte, ${ }^{6}$ F. Ledroit-Guillon, ${ }^{57}$ C. A. Lee, ${ }^{27}$ G. R. Lee, ${ }^{34 a}$ S. C. Lee, ${ }^{153}$ L. Lee, ${ }^{59}$ B. Lefebvre, ${ }^{90}$ M. Lefebvre, ${ }^{172}$ F. Legger, ${ }^{102}$ C. Leggett, ${ }^{16}$ G. Lehmann Miotto, ${ }^{32}$ W. A. Leight, ${ }^{45}$ A. Leisos, ${ }^{156, \text { hh }}$ M. A. L. Leite, ${ }^{26 \mathrm{~d}}$ R. Leitner, ${ }^{131}$ D. Lellouch, ${ }^{175}$ B. Lemmer, ${ }^{58}$ K. J. C. Leney, ${ }^{81}$ T. Lenz, ${ }^{23}$ B. Lenzi, ${ }^{32}$ R. Leone, ${ }^{7}$ S. Leone, ${ }^{126 a}$ C. Leonidopoulos, ${ }^{49}$ G. Lerner,${ }^{151}$ C. Leroy,${ }^{97}$ R. Les, ${ }^{161}$ A. A. J. Lesage, ${ }^{138}$ C. G. Lester, ${ }^{30}$ M. Levchenko, ${ }^{125}$ J. Levêque, ${ }^{5}$ D. Levin, ${ }^{92}$ L. J. Levinson, ${ }^{175}$ M. Levy, ${ }^{19}$ D. Lewis, ${ }^{79}$ B. Li,${ }^{36 a, x}$ C.-Q. Li, ${ }^{36 a}$ H. Li, ${ }^{36 \mathrm{~b}}$ L. Li ${ }^{36 \mathrm{c}}$ Q. Li,${ }^{35 \mathrm{a}, 35 \mathrm{~d}}$ Q. Li ${ }^{36 \mathrm{a}} \mathrm{S} . \mathrm{Li},{ }^{36 c, 36 \mathrm{~d}} \mathrm{X} . \mathrm{Li},{ }^{36 \mathrm{c}}$ Y. Li, ${ }^{143}$ Z. Liang, ${ }^{35 \mathrm{a}}$ B. Liberti, ${ }^{135 \mathrm{a}}$ A. Liblong, ${ }^{161}$ K. Lie, ${ }^{62 \mathrm{c}}$ A. Limosani, ${ }^{152}$ C. Y. Lin, ${ }^{30}$ K. Lin, ${ }^{93}$ S. C. Lin,${ }^{182}$ T. H. Lin, ${ }^{86}$ R. A. Linck, ${ }^{64}$ B. E. Lindquist, ${ }^{150}$ A. E. Lionti, ${ }^{52}$ E. Lipeles, ${ }^{124}$ A. Lipniacka, ${ }^{15}$ M. Lisovyi, ${ }^{60 b}$ T. M. Liss, ${ }^{169, i i}$ A. Lister, ${ }^{171}$ A. M. Litke, ${ }^{139}$ J. D. Little, ${ }^{8}$ B. Liu, ${ }^{67}$ H. Liu, ${ }^{92}$ H. Liu, ${ }^{27}$ J. K. K. Liu, ${ }^{122}$ J. B. Liu, ${ }^{36 a}$ K. Liu, ${ }^{83}$ M. Liu, ${ }^{36 a}$ P. Liu, ${ }^{16}$ Y. L. Liu, ${ }^{36 a}$ Y. Liu, ${ }^{36 a}$ M. Livan, ${ }^{123 a, 123 b}$ A. Lleres, ${ }^{57}$ J. Llorente Merino, ${ }^{35 \mathrm{a}}$ S. L. Lloyd, ${ }^{79}$ C. Y. Lo, ${ }^{62 \mathrm{~b}}$ F. Lo Sterzo, ${ }^{43}$ E. M. Lobodzinska, ${ }^{45}$ P. Loch, ${ }^{7}$ F. K. Loebinger, ${ }^{87}$ A. Loesle, ${ }^{51}$ K. M. Loew, ${ }^{25}$ T. Lohse, ${ }^{17}$ K. Lohwasser, ${ }^{141}$ M. Lokajicek, ${ }^{129}$ B. A. Long, ${ }^{24}$ J. D. Long, ${ }^{169}$ R. E. Long, ${ }^{75}$ L. Longo,${ }^{76 a, 76 b}$ K. A. Looper, ${ }^{113}$ J. A. Lopez,${ }^{34 b}$ I. Lopez Paz, ${ }^{13}$ A. Lopez Solis, ${ }^{83}$ J. Lorenz, ${ }^{102}$ N. Lorenzo Martinez, ${ }^{5}$ M. Losada, ${ }^{21}$ P. J. Lösel, ${ }^{102}$ X. Lou, ${ }^{35 a}$ A. Lounis, ${ }^{119}$ J. Love, ${ }^{6}$ P. A. Love ${ }^{75}$ H. Lu, ${ }^{62 a}$ N. Lu, ${ }^{92}$ Y. J. Lu, ${ }^{63}$ H. J. Lubattii, ${ }^{140}$ C. Luci, ${ }^{134 a, 134 b}$ A. Lucotte,${ }^{57}$ C. Luedtke, ${ }^{51}$ F. Luehring, ${ }^{64}$ I. Luise, ${ }^{83}$ W. Lukas, ${ }^{65}$ L. Luminari, ${ }^{134 a}$ B. Lund-Jensen, ${ }^{149}$ M. S. Lutz, ${ }^{89}$ P. M. Luzi, ${ }^{83}$ D. Lynn, ${ }^{27}$ R. Lysak, ${ }^{129}$ E. Lytken,${ }^{84}$ F. Lyu,${ }^{35 a}$ V. Lyubushkin, ${ }^{68}$ H. Ma, ${ }^{27}$ L. L. Ma, ${ }^{36 \mathrm{~b}}$ Y. Ma,${ }^{36 \mathrm{~b}}$ G. Maccarrone, ${ }^{50}$ A. Macchiolo, ${ }^{103}$ C. M. Macdonald, ${ }^{141}$ B. Maček ${ }^{78}$ J. Machado Miguens, ${ }^{124,128 b}$ D. Madaffari, ${ }^{170}$ R. Madar, ${ }^{37}$ W. F. Mader, ${ }^{47}$ A. Madsen, ${ }^{45}$ N. Madysa, ${ }^{47}$ J. Maeda,${ }^{70}$ S. Maeland, ${ }^{15}$ T. Maeno, ${ }^{27}$ A. S. Maevskiy, ${ }^{101}$ V. Magerl, ${ }^{51}$ C. Maidantchik, ${ }^{26 a}$ T. Maier, ${ }^{102}$ A. Maio, ${ }^{128 a, 128 b, 128 d}$ O. Majersky, ${ }^{146 a}$ S. Majewski, ${ }^{18}$ Y. Makida, ${ }^{69}$ N. Makovec, ${ }^{119}$ B. Malaescu, ${ }^{83}$ Pa. Malecki, ${ }^{42}$ V. P. Maleev, ${ }^{125}$ F. Malek,${ }^{57}$ U. Mallik, ${ }^{66}$ D. Malon, ${ }^{6}$ C. Malone, ${ }^{30}$ S. Maltezos, ${ }^{10}$ S. Malyukov, ${ }^{32}$ J. Mamuzic,${ }^{170}$ G. Mancini,${ }^{50}$ I. Mandić, ${ }^{78}$ J. Maneira, ${ }^{128 a, 128 b}$

L. Manhaes de Andrade Filho, ${ }^{26 \mathrm{~b}}$ J. Manjarres Ramos, ${ }^{47}$ K. H. Mankinen, ${ }^{84}$ A. Mann, ${ }^{102}$ A. Manousos, ${ }^{32}$ B. Mansoulie, ${ }^{138}$

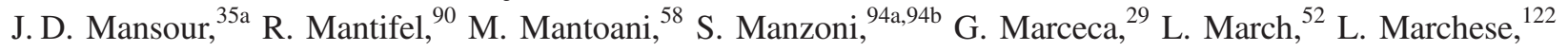
G. Marchiori, ${ }^{83}$ M. Marcisovsky, ${ }^{129}$ C. A. Marin Tobon, ${ }^{32}$ M. Marjanovic, ${ }^{37}$ D. E. Marley, ${ }^{92}$ F. Marroquim, ${ }^{26 a}$ Z. Marshall, ${ }^{16}$ M. U. F Martensson, ${ }^{168}$ S. Marti-Garcia, ${ }^{170}$ C. B. Martin, ${ }^{113}$ T. A. Martin, ${ }^{173}$ V. J. Martin, ${ }^{49}$ B. Martin dit Latour, ${ }^{15}$ M. Martinez, ${ }^{13, w}$ V. I. Martinez Outschoorn, ${ }^{89}$ S. Martin-Haugh, ${ }^{133}$ V. S. Martoiu, ${ }^{28 b}$ A. C. Martyniuk, ${ }^{81}$ A. Marzin ${ }^{32}$ L. Masetti, ${ }^{86}$ T. Mashimo, ${ }^{157}$ R. Mashinistov, ${ }^{98}$ J. Masik, ${ }^{87}$ A. L. Maslennikov, ${ }^{111, \mathrm{~d}}$ L. H. Mason, ${ }^{91}$ L. Massa, ${ }^{135 a, 135 b}$

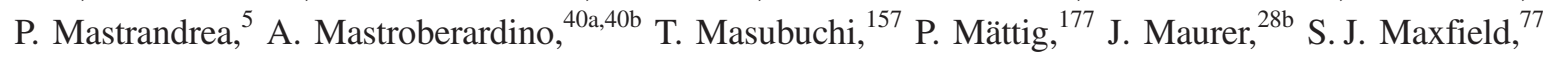

D. A. Maximov, ${ }^{111, \mathrm{~d}}$ R. Mazini, ${ }^{153}$ I. Maznas, ${ }^{156}$ S. M. Mazza, ${ }^{139}$ N. C. Mc Fadden, ${ }^{107}$ G. Mc Goldrick, ${ }^{161}$ S. P. Mc Kee, ${ }^{92}$ A. McCarn, ${ }^{92}$ T. G. McCarthy, ${ }^{103}$ L. I. McClymont, ${ }^{81}$ E. F. McDonald ${ }^{91}$ J. A. Mcfayden,${ }^{32}$ G. Mchedlidze, ${ }^{58}$ M. A. McKay, ${ }^{43}$ S. J. McMahon, ${ }^{133}$ P. C. McNamara, ${ }^{91}$ C. J. McNicol, ${ }^{173}$ R. A. McPherson, ${ }^{172,0}$ Z. A. Meadows, ${ }^{89}$ S. Meehan, ${ }^{140}$ T. J. Megy, ${ }^{51}$ S. Mehlhase, ${ }^{102}$ A. Mehta, ${ }^{77}$ T. Meideck,${ }^{57}$ K. Meier, ${ }^{60 a}$ B. Meirose, ${ }^{44}$ D. Melini, ${ }^{170, j j}$ B. R. Mellado Garcia, ${ }^{147 \mathrm{c}}$ J. D. Mellenthin,${ }^{58}$ M. Melo, ${ }^{146 \mathrm{a}}$ F. Meloni, ${ }^{18}$ A. Melzer, ${ }^{23}$ S. B. Menary,${ }^{87}$ L. Meng, ${ }^{77}$ X. T. Meng, ${ }^{92}$ A. Mengarelli, ${ }^{22 a, 22 b}$ S. Menke, ${ }^{103}$ E. Meoni, ${ }^{40 a, 40 b}$ S. Mergelmeyer, ${ }^{17}$ C. Merlassino, ${ }^{18}$ P. Mermod, ${ }^{52}$ 
L. Merola, ${ }^{106 a, 106 b}$ C. Meroni, ${ }^{94 a}$ F. S. Merritt, ${ }^{33}$ A. Messina, ${ }^{134 a, 134 b}$ J. Metcalfe, ${ }^{6}$ A. S. Mete, ${ }^{166}$ C. Meyer, ${ }^{124}$ J-P. Meyer, ${ }^{138}$ J. Meyer, ${ }^{109}$ H. Meyer Zu Theenhausen, ${ }^{60 a}$ F. Miano, ${ }^{151}$ R. P. Middleton, ${ }^{133}$ S. Miglioranzi, ${ }^{53 a, 53 b}$ L. Mijović, ${ }^{49}$ G. Mikenberg, ${ }^{175}$ M. Mikestikova, ${ }^{129}$ M. Mikuž, ${ }^{78}$ M. Milesi, ${ }^{91}$ A. Milic, ${ }^{161}$ D. A. Millar, ${ }^{79}$ D. W. Miller, ${ }^{33}$ A. Milov, ${ }^{175}$ D. A. Milstead ${ }^{148 a, 148 b}$ A. A. Minaenko, ${ }^{132}$ I. A. Minashvili,${ }^{54 b}$ A. I. Mincer,${ }^{12}$ B. Mindur,${ }^{41 \mathrm{a}}$ M. Mineev,${ }^{68}$ Y. Minegishi, ${ }^{157}$ Y. Ming, ${ }^{176}$ L. M. Mir ${ }^{13}$ A. Mirto, ${ }^{76 a, 76 b}$ K. P. Mistry, ${ }^{124}$ T. Mitani, ${ }^{174}$ J. Mitrevski, ${ }^{102}$ V. A. Mitsou, ${ }^{170}$ A. Miucci, ${ }^{18}$ P. S. Miyagawa, ${ }^{141}$ A. Mizukami, ${ }^{69}$ J. U. Mjörnmark, ${ }^{84}$ T. Mkrtchyan, ${ }^{180}$ M. Mlynarikova, ${ }^{131}$ T. Moa, ${ }^{148 a, 148 b}$ K. Mochizuki, ${ }^{97}$ P. Mogg,${ }^{51}$ S. Mohapatra, ${ }^{38}$ S. Molander, ${ }^{148 a, 148 b}$ R. Moles-Valls, ${ }^{23}$ M. C. Mondragon, ${ }^{93}$ K. Mönig,${ }^{45}$ J. Monk, ${ }^{39}$ E. Monnier, ${ }^{88}$ A. Montalbano, ${ }^{144}$ J. Montejo Berlingen, ${ }^{32}$ F. Monticelli, ${ }^{74}$ S. Monzani, ${ }^{94 a}$ R. W. Moore, ${ }^{3}$ N. Morange, ${ }^{119}$ D. Moreno, ${ }^{21}$ M. Moreno Llácer, ${ }^{32}$ P. Morettini, ${ }^{53 a}$ M. Morgenstern, ${ }^{109}$ S. Morgenstern, ${ }^{32}$ D. Mori, ${ }^{144}$ T. Mori, ${ }^{157}$ M. Morii ${ }^{59}$ M. Morinaga ${ }^{174}$ V. Morisbak, ${ }^{121}$ A. K. Morley, ${ }^{32}$ G. Mornacchi, ${ }^{32}$ J. D. Morris, ${ }^{79}$ L. Morvaj, ${ }^{150}$ P. Moschovakos, ${ }^{10}$ M. Mosidze,${ }^{54 b}$ H. J. Moss, ${ }^{141}$ J. Moss, ${ }^{145, k k}$ K. Motohashi, ${ }^{159}$ R. Mount, ${ }^{145}$ E. Mountricha, ${ }^{27}$ E. J. W. Moyse, ${ }^{89}$ S. Muanza, ${ }^{88}$ F. Mueller, ${ }^{103}$ J. Mueller, ${ }^{127}$ R. S. P. Mueller, ${ }^{102}$ D. Muenstermann, ${ }^{75}$ P. Mullen, ${ }^{56}$ G. A. Mullier ${ }^{18}$ F. J. Munoz Sanchez, ${ }^{87}$ P. Murin, ${ }^{146 \mathrm{~b}}$ W. J. Murray,${ }^{173,133}$ A. Murrone, ${ }^{94 a, 94 b}$ M. Muškinja ${ }^{78}$ C. Mwewa, ${ }^{147 a}$ A. G. Myagkov, ${ }^{132,11}$ J. Myers, ${ }^{118}$ M. Myska ${ }^{130}$ B. P. Nachman, ${ }^{16}$ O. Nackenhorst ${ }^{46}$ K. Nagai, ${ }^{122}$ R. Nagai, ${ }^{69, f f}$ K. Nagano, ${ }^{69}$ Y. Nagasaka, ${ }^{61}$ K. Nagata, ${ }^{164}$ M. Nagel,${ }^{51}$ E. Nagy, ${ }^{88}$ A. M. Nairz,${ }^{32}$ Y. Nakahama, ${ }^{105}$ K. Nakamura, ${ }^{69}$ T. Nakamura, ${ }^{157}$ I. Nakano, ${ }^{114}$ R. F. Naranjo Garcia ${ }^{45}$ R. Narayan, ${ }^{11}$ D. I. Narrias Villar, ${ }^{60 a}$ I. Naryshkin, ${ }^{125}$ T. Naumann, ${ }^{45}$ G. Navarro, ${ }^{21}$ R. Nayyar, ${ }^{7}$ H. A. Neal, ${ }^{92}$ P. Yu. Nechaeva, ${ }^{98}$ T. J. Neep, ${ }^{138}$ A. Negri, ${ }^{123 a, 123 b}$ M. Negrini, ${ }^{22 a}$ S. Nektarijevic, ${ }^{108}$ C. Nellist,${ }^{58}$ M. E. Nelson, ${ }^{122}$ S. Nemecek, ${ }^{129}$ P. Nemethy, ${ }^{112}$ M. Nessi, ${ }^{32, \mathrm{~mm}}$ M. S. Neubauer, ${ }^{169}$ M. Neumann, ${ }^{177}$ P. R. Newman, ${ }^{19}$

T. Y. Ng, ${ }^{62 \mathrm{c}}$ Y. S. Ng, ${ }^{17}$ H. D. N. Nguyen, ${ }^{88}$ T. Nguyen Manh,${ }^{97}$ R. B. Nickerson, ${ }^{122}$ R. Nicolaidou, ${ }^{138}$ J. Nielsen, ${ }^{139}$

N. Nikiforou, ${ }^{11}$ V. Nikolaenko, ${ }^{132,11}$ I. Nikolic-Audit, ${ }^{83}$ K. Nikolopoulos, ${ }^{19}$ P. Nilsson, ${ }^{27}$ Y. Ninomiya, ${ }^{69}$ A. Nisati, ${ }^{134 a}$ N. Nishu, ${ }^{36 \mathrm{c}}$ R. Nisius, ${ }^{103}$ I. Nitsche, ${ }^{46}$ T. Nitta, ${ }^{174}$ T. Nobe, ${ }^{157}$ Y. Noguchi, ${ }^{71}$ M. Nomachi, ${ }^{120}$ I. Nomidis, ${ }^{31}$ M. A. Nomura, ${ }^{27}$

T. Nooney, ${ }^{79}$ M. Nordberg, ${ }^{32}$ N. Norjoharuddeen, ${ }^{122}$ T. Novak, ${ }^{78}$ O. Novgorodova, ${ }^{47}$ R. Novotny, ${ }^{130}$ M. Nozaki, ${ }^{69}$

L. Nozka, ${ }^{117}$ K. Ntekas, ${ }^{166}$ E. Nurse, ${ }^{81}$ F. Nuti, ${ }^{91}$ K. O'Connor, ${ }^{25}$ D. C. O’Neil, ${ }^{144}$ A. A. O'Rourke, ${ }^{45}$ V. O'Shea, ${ }^{56}$ F. G. Oakham, ${ }^{31, e}$ H. Oberlack, ${ }^{103}$ T. Obermann, ${ }^{23}$ J. Ocariz, ${ }^{83}$ A. Ochi, ${ }^{70}$ I. Ochoa, ${ }^{38}$ J. P. Ochoa-Ricoux, ${ }^{34 a}$ S. Oda, ${ }^{73}$ S. Odaka, ${ }^{69}$ A. Oh ${ }^{87}$ S. H. Oh ${ }^{48}$ C. C. Ohm, ${ }^{149}$ H. Ohman, ${ }^{168}$ H. Oide, ${ }^{53 a, 53 b}$ H. Okawa, ${ }^{164}$ Y. Okumura, ${ }^{157}$ T. Okuyama ${ }^{69}$ A. Olariu, ${ }^{28 b}$ L. F. Oleiro Seabra, ${ }^{128 a}$ S. A. Olivares Pino, ${ }^{34 a}$ D. Oliveira Damazio, ${ }^{27}$ J. L. Oliver, ${ }^{1}$ M. J. R. Olsson, ${ }^{33}$ A. Olszewski, ${ }^{42}$ J. Olszowska, ${ }^{42}$ A. Onofre, ${ }^{128 a, 128 e}$ K. Onogi, ${ }^{105}$ P. U. E. Onyisi, ${ }^{11, n n}$ H. Oppen, ${ }^{121}$ M. J. Oreglia, ${ }^{33}$ Y. Oren, ${ }^{155}$ D. Orestano, ${ }^{136 a, 136 b}$ E. C. Orgill, ${ }^{87}$ N. Orlando, ${ }^{62 b}$ R. S. Orr, ${ }^{161}$ B. Osculati, ${ }^{53 a, 53 b, a}$ R. Ospanov, ${ }^{36 a}$ G. Otero y Garzon, ${ }^{29}$ H. Otono, ${ }^{73}$ M. Ouchrif, ${ }^{137 \mathrm{~d}}$ F. Ould-Saada, ${ }^{121}$ A. Ouraou, ${ }^{138}$ K. P. Oussoren, ${ }^{109}$ Q. Ouyang, ${ }^{35 a}$ M. Owen,${ }^{56}$ R. E. Owen, ${ }^{19}$ V. E. Ozcan, ${ }^{20 a}$ N. Ozturk, ${ }^{8}$ K. Pachal, ${ }^{144}$ A. Pacheco Pages, ${ }^{13}$ L. Pacheco Rodriguez, ${ }^{138}$ C. Padilla Aranda, ${ }^{13}$ S. Pagan Griso, ${ }^{16}$ M. Paganini, ${ }^{179}$ F. Paige, ${ }^{27}$ G. Palacino, ${ }^{64}$ S. Palazzo, ${ }^{40 a, 40 b}$ S. Palestini, ${ }^{32}$ M. Palka, ${ }^{41 b}$ D. Pallin, ${ }^{37}$ E. St. Panagiotopoulou, ${ }^{10}$ I. Panagoulias, ${ }^{10}$ C. E. Pandini,${ }^{52}$ J. G. Panduro Vazquez, ${ }^{80}$ P. Pani, ${ }^{32}$ D. Pantea, ${ }^{28 b}$ L. Paolozzi, ${ }^{52}$ Th. D. Papadopoulou, ${ }^{10}$ K. Papageorgiou, ${ }^{9, \mathrm{t}}$ A. Paramonov, ${ }^{6}$ D. Paredes Hernandez, ${ }^{62 \mathrm{~b}}$ B. Parida,${ }^{36 c}$ A. J. Parker, ${ }^{75}$ M. A. Parker,${ }^{30}$ K. A. Parker, ${ }^{45}$ F. Parodi, ${ }^{53 a, 53 b}$ J. A. Parsons, ${ }^{38}$ U. Parzefall,${ }^{51}$ V. R. Pascuzzi,${ }^{161}$ J. M. Pasner, ${ }^{139}$ E. Pasqualucci, ${ }^{134 a}$ S. Passaggio, ${ }^{53 \mathrm{a}}$ Fr. Pastore,${ }^{80}$ P. Pasuwan, ${ }^{148 \mathrm{a}, 148 \mathrm{~b}}$ S. Pataraia, ${ }^{86}$ J. R. Pater, ${ }^{87}$ T. Pauly, ${ }^{32}$ B. Pearson, ${ }^{103}$ S. Pedraza Lopez,${ }^{170}$ R. Pedro, ${ }^{128 a, 128 b}$ S. V. Peleganchuk, ${ }^{111, d}$ O. Penc, ${ }^{129}$ C. Peng, ${ }^{35 a, 35 \mathrm{~d}}$ H. Peng, ${ }^{36 a}$ J. Penwell, ${ }^{64}$ B. S. Peralva, ${ }^{26 \mathrm{~b}}$ M. M. Perego, ${ }^{138}$ D. V. Perepelitsa, ${ }^{27}$ F. Peri, ${ }^{17}$ L. Perini,${ }^{94 a, 94 b}$ H. Pernegger, ${ }^{32}$ S. Perrella, ${ }^{106 a, 106 b}$ V. D. Peshekhonov, ${ }^{68, a}$ K. Peters, ${ }^{45}$ R. F. Y. Peters,${ }^{87}$ B. A. Petersen, ${ }^{32}$ T. C. Petersen,${ }^{39}$ E. Petit, ${ }^{57}$ A. Petridis, ${ }^{1}$ C. Petridou, ${ }^{156}$ P. Petroff, ${ }^{119}$ E. Petrolo,${ }^{134 \mathrm{a}}$ M. Petrov, ${ }^{122}$ F. Petrucci, ${ }^{136 \mathrm{a}, 136 \mathrm{~b}}$ N. E. Pettersson, ${ }^{89}$ A. Peyaud, ${ }^{138}$ R. Pezoa, ${ }^{3 \mathrm{~b}}$ T. Pham, ${ }^{91}$ F. H. Phillips, ${ }^{93}$ P. W. Phillips, ${ }^{133}$ G. Piacquadio, ${ }^{150}$ E. Pianori, ${ }^{173}$ A. Picazio, ${ }^{89}$ M. A. Pickering, ${ }^{122}$ R. Piegaia, ${ }^{29}$ J. E. Pilcher, ${ }^{33}$ A. D. Pilkington, ${ }^{87}$ M. Pinamonti, ${ }^{135 a, 135 b}$ J. L. Pinfold, ${ }^{3}$ M. Pitt,,${ }^{175}$ M.-A. Pleier, ${ }^{27}$ V. Pleskot, ${ }^{131}$ E. Plotnikova,${ }^{68}$ D. Pluth,${ }^{67}$ P. Podberezko, ${ }^{111}$ R. Poettgen, ${ }^{84}$ R. Poggi,${ }^{123 a, 123 b}$ L. Poggioli, ${ }^{119}$ I. Pogrebnyak, ${ }^{93}$ D. Pohl, ${ }^{23}$ I. Pokharel,${ }^{58}$ G. Polesello, ${ }^{123 a}$ A. Poley, ${ }^{45}$ A. Policicchio, ${ }^{40 a, 40 b}$ R. Polifka, ${ }^{32}$ A. Polini, ${ }^{22 a}$ C. S. Pollard, ${ }^{45}$

V. Polychronakos, ${ }^{27}$ D. Ponomarenko, ${ }^{100}$ L. Pontecorvo, ${ }^{134 a}$ G. A. Popeneciu, ${ }^{28 d}$ D. M. Portillo Quintero, ${ }^{83}$ S. Pospisil, ${ }^{130}$ K. Potamianos, ${ }^{45}$ I. N. Potrap,${ }^{68}$ C. J. Potter,${ }^{30}$ H. Potti, ${ }^{11}$ T. Poulsen,${ }^{84}$ J. Poveda, ${ }^{32}$ M. E. Pozo Astigarraga, ${ }^{32}$ P. Pralavorio, ${ }^{88}$ S. Prell, ${ }^{67}$ D. Price,${ }^{87}$ M. Primavera, ${ }^{76 a}$ S. Prince,${ }^{90}$ N. Proklova, ${ }^{100}$ K. Prokofiev,${ }^{6 \mathrm{c}}$ F. Prokoshin,${ }^{34 \mathrm{~b}}$ S. Protopopescu, ${ }^{27}$ J. Proudfoot, ${ }^{6}$ M. Przybycien, ${ }^{41 a}$ A. Puri, ${ }^{169}$ P. Puzo, ${ }^{119}$ J. Qian, ${ }^{92}$ Y. Qin, ${ }^{87}$ A. Quadt,${ }^{58}$ M. Queitsch-Maitland, ${ }^{45}$ A. Qureshi, ${ }^{1}$ V. Radeka, ${ }^{27}$ S. K. Radhakrishnan, ${ }^{150}$ P. Rados,${ }^{91}$ F. Ragusa, ${ }^{94 a, 94 b}$ G. Rahal, ${ }^{181}$ J. A. Raine, ${ }^{87}$ S. Rajagopalan, ${ }^{27}$ T. Rashid, ${ }^{119}$ S. Raspopov, ${ }^{5}$ M. G. Ratti, ${ }^{94 a, 94 b}$ D. M. Rauch, ${ }^{45}$ F. Rauscher,${ }^{102}$ S. Rave, ${ }^{86}$ 
I. Ravinovich, ${ }^{175}$ J. H. Rawling, ${ }^{87}$ M. Raymond, ${ }^{32}$ A. L. Read, ${ }^{121}$ N. P. Readioff, ${ }^{57}$ M. Reale,${ }^{76 a, 76 b}$ D. M. Rebuzzi, ${ }^{123 a, 123 b}$ A. Redelbach, ${ }^{178}$ G. Redlinger, ${ }^{27}$ R. Reece, ${ }^{139}$ R. G. Reed,${ }^{147 \mathrm{c}}$ K. Reeves, ${ }^{44}$ L. Rehnisch, ${ }^{17}$ J. Reichert, ${ }^{124}$ A. Reiss ${ }^{86}$ C. Rembser ${ }^{32}$ H. Ren, ${ }^{35 a, 35 d}$ M. Rescigno, ${ }^{134 a}$ S. Resconi, ${ }^{94 a}$ E. D. Resseguie, ${ }^{124}$ S. Rettie, ${ }^{171}$ E. Reynolds, ${ }^{19}$ O. L. Rezanova, ${ }^{111, \mathrm{~d}}$ P. Reznicek, ${ }^{131}$ R. Richter, ${ }^{103}$ S. Richter, ${ }^{81}$ E. Richter-Was, ${ }^{41 \mathrm{~b}}$ O. Ricken, ${ }^{23}$ M. Ridel, ${ }^{83}$ P. Rieck, ${ }^{103}$ C. J. Riegel, ${ }^{177}$ O. Rifki, ${ }^{45}$ M. Rijssenbeek, ${ }^{150}$ A. Rimoldi, ${ }^{123 a, 123 b}$ M. Rimoldi, ${ }^{18}$ L. Rinaldi, ${ }^{22 a}$ G. Ripellino, ${ }^{149}$ B. Ristić,,${ }^{32}$ E. Ritsch, ${ }^{32}$ I. Riu, ${ }^{13}$ J. C. Rivera Vergara, ${ }^{34 a}$ F. Rizatdinova, ${ }^{116}$ E. Rizvi, ${ }^{79}$ C. Rizzi, ${ }^{13}$ R. T. Roberts,${ }^{87}$ S. H. Robertson, ${ }^{90,0}$ A. Robichaud-Veronneau ${ }^{90}$ D. Robinson,${ }^{30}$ J. E. M. Robinson, ${ }^{45}$ A. Robson,${ }^{56}$ E. Rocco,${ }^{86}$ C. Roda, ${ }^{126 a, 126 b}$ Y. Rodina,${ }^{88,00}$ S. Rodriguez Bosca, ${ }^{170}$ A. Rodriguez Perez, ${ }^{13}$ D. Rodriguez Rodriguez,${ }^{170}$ A. M. Rodríguez Vera, ${ }^{163 b}$ S. Roe, ${ }^{32}$ C. S. Rogan, ${ }^{59}$ O. Røhne, ${ }^{121}$ R. Röhrig, ${ }^{103}$ J. Roloff, ${ }^{59}$ A. Romaniouk, ${ }^{100}$ M. Romano, ${ }^{22 a, 22 b}$ S. M. Romano Saez, ${ }^{37}$ E. Romero Adam, ${ }^{170}$ N. Rompotis,${ }^{77}$ M. Ronzani, ${ }^{51}$ L. Roos, ${ }^{83}$ S. Rosati, ${ }^{134 a}$ K. Rosbach, ${ }^{51}$ P. Rose, ${ }^{139}$ N.-A. Rosien, ${ }^{58}$ E. Rossi, ${ }^{106 a, 106 b}$ L. P. Rossi, ${ }^{53 \mathrm{a}}$ L. Rossini, ${ }^{94 \mathrm{a}, 94 \mathrm{~b}}$ J. H. N. Rosten, ${ }^{30}$ R. Rosten, ${ }^{140}$ M. Rotaru, ${ }^{28 \mathrm{~b}}$ J. Rothberg, ${ }^{140}$ D. Rousseau, ${ }^{119}$ D. Roy, ${ }^{147 c}$ A. Rozanov, ${ }^{88}$ Y. Rozen, ${ }^{154}$ X. Ruan, ${ }^{147 c}$ F. Rubbo, ${ }^{145}$ F. Rühr, ${ }^{51}$ A. Ruiz-Martinez, ${ }^{31}$ Z. Rurikova, ${ }^{51}$ N. A. Rusakovich, ${ }^{68}$ H. L. Russell, ${ }^{90}$ J. P. Rutherfoord, ${ }^{7}$ N. Ruthmann, ${ }^{32}$ E. M. Rüttinger, ${ }^{45}$ Y. F. Ryabov ${ }^{125}$ M. Rybar, ${ }^{169}$ G. Rybkin, ${ }^{119}$ S. Ryu, ${ }^{6}$ A. Ryzhov, ${ }^{132}$ G. F. Rzehorz, ${ }^{58}$ A. F. Saavedra, ${ }^{152}$ G. Sabato, ${ }^{109}$ S. Sacerdoti, ${ }^{119}$ H. F-W. Sadrozinski, ${ }^{139}$ R. Sadykov, ${ }^{68}$ F. Safai Tehrani, ${ }^{134 a}$ P. Saha, ${ }^{110}$ M. Sahinsoy, ${ }^{60 a}$ M. Saimpert, ${ }^{45}$ M. Saito, ${ }^{157}$ T. Saito, ${ }^{157}$ H. Sakamoto, ${ }^{157}$ G. Salamanna, ${ }^{136 a, 136 b}$ J. E. Salazar Loyola, ${ }^{34 b}$ D. Salek, ${ }^{109}$ P. H. Sales De Bruin, ${ }^{168}$ D. Salihagic, ${ }^{103}$ A. Salnikov, ${ }^{145}$ J. Salt, ${ }^{170}$ D. Salvatore, ${ }^{40 \mathrm{a}, 40 \mathrm{~b}}$ F. Salvatore, ${ }^{151}$ A. Salvucci, ${ }^{62 \mathrm{a}, 62 \mathrm{~b}, 62 \mathrm{c}}$ A. Salzburger ${ }^{32}$

D. Sammel, ${ }^{51}$ D. Sampsonidis, ${ }^{156}$ D. Sampsonidou, ${ }^{156}$ J. Sánchez,${ }^{170}$ A. Sanchez Pineda, ${ }^{167 a, 167 c}$ H. Sandaker, ${ }^{121}$ C. O. Sander, ${ }^{45}$ M. Sandhoff, ${ }^{177}$ C. Sandoval, ${ }^{21}$ D. P. C. Sankey, ${ }^{133}$ M. Sannino, ${ }^{53 a, 53 b}$ Y. Sano, ${ }^{105}$ A. Sansoni, ${ }^{50}$ C. Santoni,${ }^{37}$ H. Santos, ${ }^{128 a}$ I. Santoyo Castillo, ${ }^{151}$ A. Sapronov, ${ }^{68}$ J. G. Saraiva, ${ }^{128 a, 128 d}$ O. Sasaki, ${ }^{69}$ K. Sato, ${ }^{164}$ E. Sauvan, ${ }^{5}$ P. Savard, ${ }^{161, e}$ N. Savic, ${ }^{103}$ R. Sawada, ${ }^{157}$ C. Sawyer, ${ }^{133}$ L. Sawyer, ${ }^{82, v}$ C. Sbarra, ${ }^{22 a}$ A. Sbrizzi, ${ }^{22 a, 22 b}$ T. Scanlon, ${ }^{81}$ D. A. Scannicchio, ${ }^{166}$ J. Schaarschmidt, ${ }^{140}$ P. Schacht, ${ }^{103}$ B. M. Schachtner, ${ }^{102}$ D. Schaefer, ${ }^{33}$ L. Schaefer, ${ }^{124}$ J. Schaeffer,${ }^{86}$ S. Schaepe ${ }^{32}$ U. Schäfer, ${ }^{86}$ A. C. Schaffer, ${ }^{119}$ D. Schaile, ${ }^{102}$ R. D. Schamberger, ${ }^{150}$ V. A. Schegelsky, ${ }^{125}$ D. Scheirich, ${ }^{131}$ F. Schenck, ${ }^{17}$ M. Schernau, ${ }^{166}$ C. Schiavi, ${ }^{53,53 b}$ S. Schier, ${ }^{139}$ L. K. Schildgen, ${ }^{23}$ Z. M. Schillaci, ${ }^{25}$ C. Schillo, ${ }^{51}$ E. J. Schioppa, ${ }^{32}$ M. Schioppa ${ }^{40 a, 40 b}$ K. E. Schleicher ${ }^{51}$ S. Schlenker, ${ }^{32}$ K. R. Schmidt-Sommerfeld, ${ }^{103}$ K. Schmieden, ${ }^{32}$ C. Schmitt, ${ }^{86}$ S. Schmitt, ${ }^{45}$ S. Schmitz, ${ }^{86}$ U. Schnoor, ${ }^{51}$ L. Schoeffel, ${ }^{138}$ A. Schoening, ${ }^{60 b}$ E. Schopf, ${ }^{23}$ M. Schott, ${ }^{86}$

J. F. P. Schouwenberg, ${ }^{108}$ J. Schovancova, ${ }^{32}$ S. Schramm, ${ }^{52}$ N. Schuh,${ }^{86}$ A. Schulte, ${ }^{86}$ H.-C. Schultz-Coulon, ${ }^{60 a}$ M. Schumacher, ${ }^{51}$ B. A. Schumm, ${ }^{139}$ Ph. Schune, ${ }^{138}$ A. Schwartzman, ${ }^{145}$ T. A. Schwarz,${ }^{92}$ H. Schweiger, ${ }^{87}$ Ph. Schwemling, ${ }^{138}$ R. Schwienhorst, ${ }^{93}$ J. Schwindling, ${ }^{138}$ A. Sciandra, ${ }^{23}$ G. Sciolla, ${ }^{25}$ M. Scornajenghi, ${ }^{40,40 b}$ F. Scuri, ${ }^{126 a}$ F. Scutti, ${ }^{91}$ L. M. Scyboz, ${ }^{103}$ J. Searcy, ${ }^{92}$ P. Seema, ${ }^{23}$ S. C. Seidel, ${ }^{107}$ A. Seiden, ${ }^{139}$ J. M. Seixas, ${ }^{26 a}$ G. Sekhniaidze, ${ }^{106 a}$ K. Sekhon, ${ }^{92}$ S. J. Sekula, ${ }^{43}$ N. Semprini-Cesari, ${ }^{22 a, 22 b}$ S. Senkin, ${ }^{37}$ C. Serfon, ${ }^{121}$ L. Serin, ${ }^{119}$ L. Serkin,,${ }^{167 a, 167 b}$ M. Sessa, ${ }^{136 a, 136 b}$ H. Severini, ${ }^{115}$ T. Šfiligoj, ${ }^{78}$ F. Sforza, ${ }^{165}$ A. Sfyrla, ${ }^{52}$ E. Shabalina, ${ }^{58}$ J. D. Shahinian, ${ }^{139}$ N. W. Shaikh, ${ }^{148 a, 148 b}$ L. Y. Shan, ${ }^{35 a}$ R. Shang, ${ }^{169}$ J. T. Shank, ${ }^{24}$ M. Shapiro, ${ }^{16}$ A. S. Sharma, ${ }^{1}$ P. B. Shatalov, ${ }^{99}$ K. Shaw, ${ }^{167 a, 167 b}$ S. M. Shaw, ${ }^{87}$ A. Shcherbakova, ${ }^{148 a, 148 b}$ C. Y. Shehu, ${ }^{151}$ Y. Shen, ${ }^{115}$ N. Sherafati, ${ }^{31}$ A. D. Sherman, ${ }^{24}$ P. Sherwood, ${ }^{81}$ L. Shi, ${ }^{153, p p}$ S. Shimizu,${ }^{70}$ C. O. Shimmin, ${ }^{179}$ M. Shimojima, ${ }^{104}$ I. P. J. Shipsey, ${ }^{122}$ S. Shirabe, ${ }^{73}$ M. Shiyakova, ${ }^{68, q q}$ J. Shlomi, ${ }^{175}$ A. Shmeleva, ${ }^{98}$ D. Shoaleh Saadi, ${ }^{97}$ M. J. Shochet, ${ }^{33}$ S. Shojaii, ${ }^{91}$ D. R. Shope,${ }^{115}$ S. Shrestha, ${ }^{113}$ E. Shulga ${ }^{100}$ P. Sicho, ${ }^{129}$ A. M. Sickles, ${ }^{169}$ P. E. Sidebo,${ }^{149}$ E. Sideras Haddad,${ }^{147 c}$ O. Sidiropoulou, ${ }^{178}$ A. Sidoti, ${ }^{22 a, 22 b}$ F. Siegert, ${ }^{47}$ Dj. Sijacki, ${ }^{14}$ J. Silva, ${ }^{128 a, 128 d}$ M. Silva Jr., ${ }^{176}$ S. B. Silverstein, ${ }^{148 a}$ L. Simic,${ }^{68}$ S. Simion, ${ }^{119}$ E. Simioni, ${ }^{86}$ B. Simmons, ${ }^{81}$ M. Simon, ${ }^{86}$ P. Sinervo, ${ }^{161}$ N. B. Sinev, ${ }^{118}$ M. Sioli, ${ }^{22 a, 22 b}$ G. Siragusa, ${ }^{178}$ I. Siral,${ }^{92}$ S. Yu. Sivoklokov, ${ }^{101}$ J. Sjölin, ${ }^{148 a, 148 b}$ M. B. Skinner, ${ }^{75}$ P. Skubic, ${ }^{115}$ M. Slater, ${ }^{19}$ T. Slavicek, ${ }^{130}$ M. Slawinska, ${ }^{42}$ K. Sliwa, ${ }^{165}$ R. Slovak, ${ }^{131}$ V. Smakhtin, ${ }^{175}$ B. H. Smart, ${ }^{5}$ J. Smiesko, ${ }^{146 a}$ N. Smirnov, ${ }^{100}$ S. Yu. Smirnov, ${ }^{100}$ Y. Smirnov, ${ }^{100}$ L. N. Smirnova, ${ }^{101, r r}$ O. Smirnova, ${ }^{84}$ J. W. Smith, ${ }^{58}$ M. N. K. Smith, ${ }^{38}$ R. W. Smith, ${ }^{38}$ M. Smizanska, ${ }^{75}$ K. Smolek, ${ }^{130}$ A. A. Snesarev, ${ }^{98}$ I. M. Snyder, ${ }^{118}$ S. Snyder,${ }^{27}$ R. Sobie, ${ }^{172,0}$ F. Socher, ${ }^{47}$ A. M. Soffa, ${ }^{166}$ A. Soffer,${ }^{155}$ A. Søgaard, ${ }^{49}$ D. A. Soh, ${ }^{153}$ G. Sokhrannyi, ${ }^{78}$ C. A. Solans Sanchez,${ }^{32}$ M. Solar, ${ }^{130}$ E. Yu. Soldatov, ${ }^{100}$ U. Soldevila, ${ }^{170}$ A. A. Solodkov, ${ }^{132}$ A. Soloshenko, ${ }^{68}$ O. V. Solovyanov ${ }^{132}$ V. Solovyev, ${ }^{125}$ P. Sommer, ${ }^{141}$ H. Son, ${ }^{165}$ W. Song, ${ }^{133}$ A. Sopczak,${ }^{130}$ F. Sopkova, ${ }^{146 b}$

D. Sosa, ${ }^{60 b}$ C. L. Sotiropoulou, ${ }^{126 a, 126 b}$ S. Sottocornola, ${ }^{123 a, 123 b}$ R. Soualah, ${ }^{167 a, 167 c}$ A. M. Soukharev, ${ }^{111, d}$ D. South, ${ }^{45}$ B. C. Sowden, ${ }^{80}$ S. Spagnolo, ${ }^{76 a, 76 b}$ M. Spalla, ${ }^{103}$ M. Spangenberg, ${ }^{173}$ F. Spanò,${ }^{80}$ D. Sperlich, ${ }^{17}$ F. Spettel, ${ }^{103}$ T. M. Spieker ${ }^{60 a}$ R. Spighi, ${ }^{22 a}$ G. Spigo, ${ }^{32}$ L. A. Spiller, ${ }^{91}$ M. Spousta, ${ }^{131}$ R. D. St. Denis, ${ }^{56, a}$ A. Stabile, ${ }^{94 a, 94 b}$ R. Stamen, ${ }^{60 a}$ S. Stamm, ${ }^{17}$ E. Stanecka, ${ }^{42}$ R. W. Stanek, ${ }^{6}$ C. Stanescu, ${ }^{136 a}$ M. M. Stanitzki, ${ }^{45}$ B. S. Stapf, ${ }^{109}$ S. Stapnes, ${ }^{121}$ 
E. A. Starchenko, ${ }^{132}$ G. H. Stark, ${ }^{33}$ J. Stark,${ }^{57}$ S. H Stark, ${ }^{39}$ P. Staroba, ${ }^{129}$ P. Starovoitov, ${ }^{60 a}$ S. Stärz, ${ }^{32}$ R. Staszewski, ${ }^{42}$ M. Stegler ${ }^{45}$ P. Steinberg, ${ }^{27}$ B. Stelzer, ${ }^{144}$ H. J. Stelzer, ${ }^{32}$ O. Stelzer-Chilton, ${ }^{163 a}$ H. Stenzel, ${ }^{55}$ T. J. Stevenson, ${ }^{79}$ G. A. Stewart, ${ }^{32}$ M. C. Stockton, ${ }^{118}$ G. Stoicea, ${ }^{28 b}$ P. Stolte,${ }^{58}$ S. Stonjek, ${ }^{103}$ A. Straessner, ${ }^{47}$ M. E. Stramaglia, ${ }^{18}$ J. Strandberg, ${ }^{149}$ S. Strandberg, ${ }^{148 a, 148 b}$ M. Strauss, ${ }^{115}$ P. Strizenec, ${ }^{146 b}$ R. Ströhmer, ${ }^{178}$ D. M. Strom, ${ }^{118}$ R. Stroynowski, ${ }^{43}$ A. Strubig, ${ }^{49}$ S. A. Stucci, ${ }^{27}$ B. Stugu, ${ }^{15}$ N. A. Styles, ${ }^{45}$ D. Su, ${ }^{145}$ J. Su, ${ }^{127}$ S. Suchek, ${ }^{60 a}$ Y. Sugaya,${ }^{120}$ M. Suk, ${ }^{130}$ V. V. Sulin, ${ }^{98}$ DMS Sultan, ${ }^{52}$ S. Sultansoy,${ }^{4 c}$ T. Sumida, ${ }^{71}$ S. Sun, ${ }^{92}$ X. Sun, ${ }^{3}$ K. Suruliz, ${ }^{151}$ C. J. E. Suster,${ }^{152}$ M. R. Sutton, ${ }^{151}$ S. Suzuki, ${ }^{69}$ M. Svatos, ${ }^{129}$ M. Swiatlowski, ${ }^{33}$ S. P. Swift, ${ }^{2}$ A. Sydorenko, ${ }^{86}$ I. Sykora, ${ }^{146 a}$ T. Sykora, ${ }^{131}$ D. Ta, ${ }^{86}$ K. Tackmann, ${ }^{45}$ J. Taenzer, ${ }^{155}$ A. Taffard,${ }^{166}$ R. Tafirout, ${ }^{163 a}$ E. Tahirovic, ${ }^{79}$ N. Taiblum, ${ }^{155}$ H. Takai, ${ }^{27}$ R. Takashima, ${ }^{72}$ E. H. Takasugi, ${ }^{103}$ K. Takeda, ${ }^{70}$ T. Takeshita, ${ }^{142}$ Y. Takubo, ${ }^{69}$ M. Talby, ${ }^{88}$ A. A. Talyshev, ${ }^{11, d}$ J. Tanaka, ${ }^{157}$ M. Tanaka, ${ }^{159}$ R. Tanaka, ${ }^{119}$ R. Tanioka, ${ }^{70}$ B. B. Tannenwald, ${ }^{113}$ S. Tapia Araya, ${ }^{34 b}$ S. Tapprogge, ${ }^{86}$ A. T. Tarek Abouelfadl Mohamed, ${ }^{83}$ S. Tarem, ${ }^{154}$ G. Tarna,${ }^{28 b, q}$ G. F. Tartarelli, ${ }^{94 a}$ P. Tas, ${ }^{131}$ M. Tasevsky, ${ }^{129}$ T. Tashiro, ${ }^{71}$ E. Tassi, ${ }^{40 a, 40 b}$

A. Tavares Delgado, ${ }^{128 a, 128 b}$ Y. Tayalati, ${ }^{137 \mathrm{e}}$ A. C. Taylor,${ }^{107}$ A. J. Taylor, ${ }^{49}$ G. N. Taylor, ${ }^{91}$ P. T. E. Taylor, ${ }^{91}$ W. Taylor, ${ }^{163 b}$ P. Teixeira-Dias, ${ }^{80}$ D. Temple, ${ }^{144}$ H. Ten Kate, ${ }^{32}$ P. K. Teng, ${ }^{153}$ J. J. Teoh, ${ }^{120}$ F. Tepel, ${ }^{177}$ S. Terada, ${ }^{69}$ K. Terashi, ${ }^{157}$ J. Terron, ${ }^{85}$ S. Terzo, ${ }^{13}$ M. Testa, ${ }^{50}$ R. J. Teuscher,${ }^{161, o}$ S. J. Thais, ${ }^{179}$ T. Theveneaux-Pelzer, ${ }^{45}$ F. Thiele, ${ }^{39}$ J. P. Thomas, ${ }^{19}$ P. D. Thompson, ${ }^{19}$ A. S. Thompson,${ }^{56}$ L. A. Thomsen, ${ }^{179}$ E. Thomson, ${ }^{124}$ Y. Tian, ${ }^{38}$ R. E. Ticse Torres, ${ }^{58}$

V. O. Tikhomirov, ${ }^{98, s s}$ Yu. A. Tikhonov, ${ }^{111, \mathrm{~d}}$ S. Timoshenko, ${ }^{100}$ P. Tipton, ${ }^{179}$ S. Tisserant, ${ }^{88}$ K. Todome,${ }^{159}$

S. Todorova-Nova, ${ }^{5}$ S. Todt, ${ }^{47}$ J. Tojo, ${ }^{73}$ S. Tokár, ${ }^{146 a}$ K. Tokushuku, ${ }^{69}$ E. Tolley, ${ }^{113}$ M. Tomoto, ${ }^{105}$ L. Tompkins, ${ }^{145, t t}$ K. Toms, ${ }^{107}$ B. Tong, ${ }^{59}$ P. Tornambe, ${ }^{51}$ E. Torrence, ${ }^{118}$ H. Torres, ${ }^{47}$ E. Torró Pastor, ${ }^{140}$ J. Toth,,${ }^{88, \text { uu }}$ F. Touchard, ${ }^{88}$ D. R. Tovey, ${ }^{141}$ C. J. Treado, ${ }^{112}$ T. Trefzger, ${ }^{178}$ F. Tresoldi, ${ }^{151}$ A. Tricoli, ${ }^{27}$ I. M. Trigger, ${ }^{163 a}$ S. Trincaz-Duvoid ${ }^{83}$ M. F. Tripiana, ${ }^{13}$ W. Trischuk, ${ }^{161}$ B. Trocmé, ${ }^{57}$ A. Trofymov, ${ }^{45}$ C. Troncon, ${ }^{94 a}$ M. Trovatelli, ${ }^{172}$ L. Truong, ${ }^{147 b}$ M. Trzebinski, ${ }^{42}$ A. Trzupek, ${ }^{42}$ K. W. Tsang, ${ }^{62 \mathrm{a}}$ J. C-L. Tseng, ${ }^{122}$ P. V. Tsiareshka, ${ }^{95}$ N. Tsirintanis, ${ }^{9}$ S. Tsiskaridze, ${ }^{13}$

V. Tsiskaridze, ${ }^{150}$ E. G. Tskhadadze, ${ }^{54 \mathrm{a}}$ I. I. Tsukerman, ${ }^{99}$ V. Tsulaia, ${ }^{16}$ S. Tsuno, ${ }^{69}$ D. Tsybychev, ${ }^{150}$ Y. Tu, ${ }^{62 b}$ A. Tudorache, ${ }^{28 \mathrm{~b}}$ V. Tudorache, ${ }^{28 \mathrm{~b}}$ T. T. Tulbure, ${ }^{28 \mathrm{a}}$ A. N. Tuna, ${ }^{59}$ S. Turchikhin, ${ }^{68}$ D. Turgeman, ${ }^{175}$ I. Turk Cakir, ${ }^{4 \mathrm{~b}, \mathrm{vv}}$ R. Turra ${ }^{94 a}$ P. M. Tuts ${ }^{38}$ G. Ucchielli, ${ }^{22 a, 22 b}$ I. Ueda, ${ }^{69}$ M. Ughetto, ${ }^{148 a, 148 b}$ F. Ukegawa, ${ }^{164}$ G. Unal, ${ }^{32}$ A. Undrus, ${ }^{27}$ G. Unel, ${ }^{166}$ F. C. Ungaro, ${ }^{91}$ Y. Unno, ${ }^{69}$ K. Uno, ${ }^{157}$ J. Urban,,${ }^{146 b}$ P. Urquijo, ${ }^{91}$ P. Urrejola, ${ }^{86}$ G. Usai, ${ }^{8}$ J. Usui,${ }^{69}$ L. Vacavant,${ }^{88}$ V. Vacek, ${ }^{130}$ B. Vachon, ${ }^{90}$ K. O. H. Vadla, ${ }^{121}$ A. Vaidya, ${ }^{81}$ C. Valderanis, ${ }^{102}$ E. Valdes Santurio, ${ }^{148 a, 148 b}$ M. Valente, ${ }^{52}$

S. Valentinetti, ${ }^{22 a, 22 b}$ A. Valero, ${ }^{170}$ L. Valéry, ${ }^{45}$ A. Vallier, ${ }^{5}$ J. A. Valls Ferrer, ${ }^{170}$ W. Van Den Wollenberg, ${ }^{109}$

H. van der Graaf, ${ }^{109}$ P. van Gemmeren, ${ }^{6}$ J. Van Nieuwkoop, ${ }^{144}$ I. van Vulpen, ${ }^{109}$ M. C. van Woerden, ${ }^{109}$ M. Vanadia, ${ }^{135 a, 135 b}$ W. Vandelli, ${ }^{32}$ A. Vaniachine, ${ }^{160}$ P. Vankov, ${ }^{109}$ R. Vari, ${ }^{134 a}$ E. W. Varnes, ${ }^{7}$ C. Varni, ${ }^{53 a, 53 b}$ T. Varol, ${ }^{43}$ D. Varouchas, ${ }^{119}$

A. Vartapetian, ${ }^{8}$ K. E. Varvell, ${ }^{152}$ J. G. Vasquez, ${ }^{179}$ G. A. Vasquez, ${ }^{34 b}$ F. Vazeille, ${ }^{37}$ D. Vazquez Furelos, ${ }^{13}$

T. Vazquez Schroeder, ${ }^{90}$ J. Veatch, ${ }^{58}$ L. M. Veloce, ${ }^{161}$ F. Veloso, ${ }^{128 a, 128 c}$ S. Veneziano, ${ }^{134 a}$ A. Ventura, ${ }^{76 a, 76 b}$ M. Venturi, ${ }^{172}$ N. Venturi, ${ }^{32}$ V. Vercesi, ${ }^{123 a}$ M. Verducci, ${ }^{136 a, 136 b}$ W. Verkerke, ${ }^{109}$ A. T. Vermeulen, ${ }^{109}$ J. C. Vermeulen, ${ }^{109}$ M. C. Vetterli, ${ }^{144, e}$ N. Viaux Maira, ${ }^{34 b}$ O. Viazlo, ${ }^{84}$ I. Vichou, ${ }^{169, a}$ T. Vickey, ${ }^{141}$ O. E. Vickey Boeriu, ${ }^{141}$ G. H. A. Viehhauser, ${ }^{122}$ S. Viel, ${ }^{16}$ L. Vigani, ${ }^{122}$ M. Villa, ${ }^{22 a, 22 b}$ M. Villaplana Perez,${ }^{94 a, 94 b}$ E. Vilucchi, ${ }^{50}$ M. G. Vincter, ${ }^{31}$ V. B. Vinogradov,${ }^{68}$

A. Vishwakarma ${ }^{45}$ C. Vittori, ${ }^{22 a, 22 b}$ I. Vivarelli, ${ }^{151}$ S. Vlachos,${ }^{10}$ M. Vogel,${ }^{177}$ P. Vokac, ${ }^{130}$ G. Volpi,${ }^{13}$

S. E. von Buddenbrock, ${ }^{147 c}$ E. von Toerne, ${ }^{23}$ V. Vorobel, ${ }^{131}$ K. Vorobev, ${ }^{100}$ M. Vos, ${ }^{170}$ J. H. Vossebeld,${ }^{77}$ N. Vranjes, ${ }^{14}$ M. Vranjes Milosavljevic, ${ }^{14}$ V. Vrba, ${ }^{130}$ M. Vreeswijk, ${ }^{109}$ R. Vuillermet, ${ }^{32}$ I. Vukotic, ${ }^{33}$ P. Wagner, ${ }^{23}$ W. Wagner, ${ }^{177}$

J. Wagner-Kuhr, ${ }^{102}$ H. Wahlberg, ${ }^{74}$ S. Wahrmund, ${ }^{47}$ K. Wakamiya,${ }^{70}$ J. Walder ${ }^{75}$ R. Walker, ${ }^{102}$ W. Walkowiak, ${ }^{143}$ V. Wallangen, ${ }^{148 a, 148 b}$ A. M. Wang, ${ }^{59}$ C. Wang, ${ }^{36 b, q}$ F. Wang, ${ }^{176}$ H. Wang, ${ }^{16}$ H. Wang, ${ }^{3}$ J. Wang, ${ }^{60 b}$ J. Wang, ${ }^{152}$ Q. Wang, ${ }^{115}$

R.-J. Wang, ${ }^{83}$ R. Wang, ${ }^{6}$ S. M. Wang, ${ }^{153}$ T. Wang, ${ }^{38}$ W. Wang, ${ }^{35 \mathrm{~b}}$ W. Wang, ${ }^{36 \mathrm{a}, \mathrm{ww}}$ Z. Wang, ${ }^{36 \mathrm{c}}$ C. Wanotayaroj, ${ }^{45}$

A. Warburton, ${ }^{90}$ C. P. Ward, ${ }^{30}$ D. R. Wardrope, ${ }^{81}$ A. Washbrook, ${ }^{49}$ P. M. Watkins, ${ }^{19}$ A. T. Watson, ${ }^{19}$ M. F. Watson, ${ }^{19}$

G. Watts, ${ }^{140}$ S. Watts, ${ }^{87}$ B. M. Waugh, ${ }^{81}$ A. F. Webb, ${ }^{11}$ S. Webb,${ }^{86}$ M. S. Weber, ${ }^{18}$ S. M. Weber, ${ }^{60}$ S. A. Weber, ${ }^{31}$

J. S. Webster, ${ }^{6}$ A. R. Weidberg, ${ }^{122}$ B. Weinert, ${ }^{64}$ J. Weingarten, ${ }^{58}$ M. Weirich,${ }^{86}$ C. Weiser, ${ }^{51}$ P. S. Wells,${ }^{32}$ T. Wenaus, ${ }^{27}$

T. Wengler, ${ }^{32}$ S. Wenig, ${ }^{32}$ N. Wermes, ${ }^{23}$ M. D. Werner, ${ }^{67}$ P. Werner, ${ }^{32}$ M. Wessels,${ }^{60 a}$ T. D. Weston, ${ }^{18}$ K. Whalen, ${ }^{118}$ N. L. Whallon, ${ }^{140}$ A. M. Wharton, ${ }^{75}$ A. S. White, ${ }^{92}$ A. White, ${ }^{8}$ M. J. White, ${ }^{1}$ R. White, ${ }^{34 b}$ D. Whiteson, ${ }^{166}$ B. W. Whitmore, ${ }^{75}$ F. J. Wickens, ${ }^{133}$ W. Wiedenmann, ${ }^{176}$ M. Wielers, ${ }^{133}$ C. Wiglesworth, ${ }^{39}$ L. A. M. Wiik-Fuchs, ${ }^{51}$ A. Wildauer, ${ }^{103}$ F. Wilk, ${ }^{87}$ H. G. Wilkens ${ }^{32}$ H. H. Williams, ${ }^{124}$ S. Williams, ${ }^{30}$ C. Willis, ${ }^{93}$ S. Willocq, ${ }^{89}$ J. A. Wilson, ${ }^{19}$ I. Wingerter-Seez, ${ }^{5}$

E. Winkels, ${ }^{151}$ F. Winklmeier, ${ }^{118}$ O. J. Winston, ${ }^{151}$ B. T. Winter, ${ }^{23}$ M. Wittgen, ${ }^{145}$ M. Wobisch, ${ }^{82, v}$ A. Wolf, ${ }^{86}$ T. M. H. Wolf, ${ }^{109}$ R. Wolff, ${ }^{88}$ M. W. Wolter, ${ }^{42}$ H. Wolters, ${ }^{128 a, 128 c}$ V. W. S. Wong, ${ }^{171}$ N. L. Woods, ${ }^{139}$ S. D. Worm, ${ }^{19}$ 
B. K. Wosiek, ${ }^{42}$ K. W. Wozniak, ${ }^{42}$ M. Wu, ${ }^{33}$ S. L. Wu, ${ }^{176}$ X. Wu, ${ }^{52}$ Y. Wu, ${ }^{36 a}$ T. R. Wyatt, ${ }^{87}$ B. M. Wynne, ${ }^{49}$ S. Xella, ${ }^{39}$

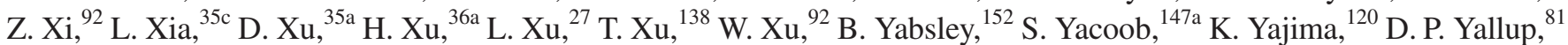
D. Yamaguchi, ${ }^{159}$ Y. Yamaguchi, ${ }^{159}$ A. Yamamoto, ${ }^{69}$ T. Yamanaka, ${ }^{157}$ F. Yamane,${ }^{70}$ M. Yamatani, ${ }^{157}$ T. Yamazaki, ${ }^{157}$ Y. Yamazaki, ${ }^{70}$ Z. Yan, ${ }^{24}$ H. Yang, ${ }^{36 c, 36 d}$ H. Yang, ${ }^{16}$ S. Yang, ${ }^{66}$ Y. Yang, ${ }^{153}$ Y. Yang, ${ }^{157}$ Z. Yang, ${ }^{15}$ W-M. Yao, ${ }^{16}$ Y. C. Yap ${ }^{45}$ Y. Yasu, ${ }^{69}$ E. Yatsenko, ${ }^{5}$ K. H. Yau Wong, ${ }^{23}$ J. Ye, ${ }^{43}$ S. Ye, ${ }^{27}$ I. Yeletskikh, ${ }^{68}$ E. Yigitbasi, ${ }^{24}$ E. Yildirim, ${ }^{86}$ K. Yorita, ${ }^{174}$ K. Yoshihara, ${ }^{124}$ C. Young, ${ }^{145}$ C. J. S. Young, ${ }^{32}$ J. Yu, ${ }^{8}$ J. Yu ${ }^{67}$ S. P. Y. Yuen, ${ }^{23}$ I. Yusuff, ${ }^{30, x x}$ B. Zabinski, ${ }^{42}$ G. Zacharis, ${ }^{10}$ R. Zaidan, ${ }^{13}$ A. M. Zaitsev, ${ }^{132,11}$ N. Zakharchuk,${ }^{45}$ J. Zalieckas, ${ }^{15}$ S. Zambito, ${ }^{59}$ D. Zanzi, ${ }^{32}$ C. Zeitnitz, ${ }^{177}$ G. Zemaityte, ${ }^{122}$ J. C. Zeng, ${ }^{169}$ Q. Zeng, ${ }^{145}$ O. Zenin, ${ }^{132}$ T. Ženiš, ${ }^{146 a}$ D. Zerwas, ${ }^{119}$ D. Zhang, ${ }^{36 b}$ D. Zhang, ${ }^{92}$ F. Zhang, ${ }^{176}$ G. Zhang, ${ }^{36 a, w w}$ H. Zhang, ${ }^{119}$ J. Zhang, ${ }^{6}$ L. Zhang, ${ }^{51}$ L. Zhang, ${ }^{36 \mathrm{a}}$ M. Zhang, ${ }^{169}$ P. Zhang, ${ }^{35 \mathrm{~b}}$ R. Zhang, ${ }^{23}$ R. Zhang, ${ }^{36 a, q}$ X. Zhang, ${ }^{36 \mathrm{~b}}$ Y. Zhang, ${ }^{35 a, 35 d}$ Z. Zhang, ${ }^{119}$ X. Zhao, ${ }^{43}$ Y. Zhao, ${ }^{36 b, y}$ Z. Zhao, ${ }^{36 a}$ A. Zhemchugov, ${ }^{68}$ B. Zhou, ${ }^{92}$ C. Zhou, ${ }^{176}$ L. Zhou, ${ }^{43}$ M. Zhou, ${ }^{35 a, 35 d}$ M. Zhou, ${ }^{150}$ N. Zhou, ${ }^{36 c}$ Y. Zhou, ${ }^{7}$ C. G. Zhu, ${ }^{36 b}$ H. Zhu, ${ }^{35 a}$ J. Zhu, ${ }^{92}$ Y. Zhu, ${ }^{36 a}$ X. Zhuang, ${ }^{35 a}$ K. Zhukov, ${ }^{98}$ V. Zhulanov, ${ }^{11, y y}$ A. Zibell, ${ }^{178}$ D. Zieminska, ${ }^{64}$ N. I. Zimine, ${ }^{68}$ S. Zimmermann, ${ }^{51}$ Z. Zinonos, ${ }^{103}$ M. Zinser, ${ }^{86}$ M. Ziolkowski, ${ }^{143}$ L. Živković, ${ }^{14}$ G. Zobernig, ${ }^{176}$ A. Zoccoli, ${ }^{22 a, 22 b}$ T. G. Zorbas, ${ }^{141}$ R. Zou, ${ }^{33}$ M. zur Nedden, ${ }^{17}$ and L. Zwalinski ${ }^{32}$

(ATLAS Collaboration)

\footnotetext{
${ }^{1}$ Department of Physics, University of Adelaide, Adelaide, Australia

${ }^{2}$ Physics Department, SUNY Albany, Albany, New York, USA

${ }^{3}$ Department of Physics, University of Alberta, Edmonton, Alberta, Canada

${ }^{4 a}$ Department of Physics, Ankara University, Ankara, Turkey

${ }^{4 \mathrm{~b}}$ Istanbul Aydin University, Istanbul, Turkey

${ }^{4 \mathrm{c}}$ Division of Physics, TOBB University of Economics and Technology, Ankara, Turkey

${ }^{5}$ LAPP, CNRS/IN2P3 and Université Savoie Mont Blanc, Annecy-le-Vieux, France

${ }^{6}$ High Energy Physics Division, Argonne National Laboratory, Argonne, Illinois, USA

${ }^{7}$ Department of Physics, University of Arizona, Tucson, Arizona, USA

${ }^{8}$ Department of Physics, The University of Texas at Arlington, Arlington, Texas, USA

${ }^{9}$ Physics Department, National and Kapodistrian University of Athens, Athens, Greece

${ }^{10}$ Physics Department, National Technical University of Athens, Zografou, Greece

${ }^{11}$ Department of Physics, The University of Texas at Austin, Austin, Texas, USA

${ }^{12}$ Institute of Physics, Azerbaijan Academy of Sciences, Baku, Azerbaijan

${ }^{13}$ Institut de Física d'Altes Energies (IFAE), The Barcelona Institute of Science and Technology, Barcelona, Spain

${ }^{14}$ Institute of Physics, University of Belgrade, Belgrade, Serbia

${ }^{15}$ Department for Physics and Technology, University of Bergen, Bergen, Norway

${ }^{16}$ Physics Division, Lawrence Berkeley National Laboratory and University of California, Berkeley, California, USA

${ }^{17}$ Department of Physics, Humboldt University, Berlin, Germany

${ }^{18}$ Albert Einstein Center for Fundamental Physics and Laboratory for High Energy Physics, University of Bern, Bern, Switzerland

${ }^{19}$ School of Physics and Astronomy, University of Birmingham, Birmingham, United Kingdom

${ }^{20 a}$ Department of Physics, Bogazici University, Istanbul, Turkey

${ }^{20 \mathrm{~b}}$ Department of Physics Engineering, Gaziantep University, Gaziantep, Turkey

${ }^{20 \mathrm{~d}}$ Istanbul Bilgi University, Faculty of Engineering and Natural Sciences, Istanbul, Turkey

${ }^{20 \mathrm{e}}$ Bahcesehir University, Faculty of Engineering and Natural Sciences, Istanbul, Turkey

${ }^{21}$ Centro de Investigaciones, Universidad Antonio Narino, Bogota, Colombia

${ }^{22 a}$ INFN Sezione di Bologna, Bologna, Italy

${ }^{22 \mathrm{~b}}$ Dipartimento di Fisica e Astronomia, Università di Bologna, Bologna, Italy

${ }^{23}$ Physikalisches Institut, University of Bonn, Bonn, Germany

${ }^{24}$ Department of Physics, Boston University, Boston, Massachusetts, USA

${ }^{25}$ Department of Physics, Brandeis University, Waltham, Massachusetts, USA

${ }^{26 a}$ Universidade Federal do Rio De Janeiro COPPE/EE/IF, Rio de Janeiro, Brazil

${ }^{26 \mathrm{~b}}$ Electrical Circuits Department, Federal University of Juiz de Fora (UFJF), Juiz de Fora, Brazil

${ }^{26 c}$ Federal University of Sao Joao del Rei (UFSJ), Sao Joao del Rei, Brazil

${ }^{26 \mathrm{~d}}$ Instituto de Fisica, Universidade de Sao Paulo, Sao Paulo, Brazil

${ }^{27}$ Physics Department, Brookhaven National Laboratory, Upton, New York, USA

${ }^{28 a}$ Transilvania University of Brasov, Brasov, Romania

${ }^{28 \mathrm{~b}}$ Horia Hulubei National Institute of Physics and Nuclear Engineering, Bucharest, Romania
} 


\footnotetext{
${ }^{28 c}$ Department of Physics, Alexandru Ioan Cuza University of Iasi, Iasi, Romania

${ }^{28 \mathrm{~d}}$ National Institute for Research and Development of Isotopic and Molecular Technologies, Physics Department, Cluj Napoca, Romania

${ }^{28 \mathrm{e}}$ University Politehnica Bucharest, Bucharest, Romania

${ }^{28 \mathrm{f}}$ West University in Timisoara, Timisoara, Romania

${ }^{29}$ Departamento de Física, Universidad de Buenos Aires, Buenos Aires, Argentina

${ }^{30}$ Cavendish Laboratory, University of Cambridge, Cambridge, United Kingdom

${ }^{31}$ Department of Physics, Carleton University, Ottawa, Ontario, Canada

${ }^{32}$ CERN, Geneva, Switzerland

${ }^{33}$ Enrico Fermi Institute, University of Chicago, Chicago, Illinois, USA

${ }^{34 a}$ Departamento de Física, Pontificia Universidad Católica de Chile, Santiago, Chile

${ }^{34 \mathrm{~b}}$ Departamento de Física, Universidad Técnica Federico Santa María, Valparaíso, Chile

${ }^{35 a}$ Institute of High Energy Physics, Chinese Academy of Sciences, Beijing, China

${ }^{35 \mathrm{~b}}$ Department of Physics, Nanjing University, Jiangsu, China

${ }^{35 \mathrm{c}}$ Physics Department, Tsinghua University, Beijing, China

${ }^{35 \mathrm{~d}}$ University of Chinese Academy of Science (UCAS), Beijing, China

${ }^{36 a}$ Department of Modern Physics and State Key Laboratory of Particle Detection and Electronics,

University of Science and Technology of China, Anhui, China

${ }^{36 \mathrm{~b}}$ School of Physics, Shandong University, Shandong, China

${ }^{36 c}$ School of Physics and Astronomy, Key Laboratory for Particle Physics, Astrophysics and Cosmology, Ministry of Education;

Shanghai Key Laboratory for Particle Physics and Cosmology, Shanghai Jiao Tong University, Shanghai, China

${ }^{36 \mathrm{~d}}$ Tsung-Dao Lee Institute, Shanghai, China

${ }^{37}$ Université Clermont Auvergne, CNRS/IN2P3, LPC, Clermont-Ferrand, France

${ }^{38}$ Nevis Laboratory, Columbia University, Irvington, New York, USA

${ }^{39}$ Niels Bohr Institute, University of Copenhagen, Kobenhavn, Denmark

${ }^{40 a}$ INFN Gruppo Collegato di Cosenza, Laboratori Nazionali di Frascati, Frascati, Italy

${ }^{40 \mathrm{~b}}$ Dipartimento di Fisica, Università della Calabria, Rende, Italy

${ }^{41 \mathrm{a}}$ AGH University of Science and Technology, Faculty of Physics and Applied Computer Science, Krakow, Poland

${ }^{41 \mathrm{~b}}$ Marian Smoluchowski Institute of Physics, Jagiellonian University, Krakow, Poland

${ }^{42}$ Institute of Nuclear Physics Polish Academy of Sciences, Krakow, Poland

${ }^{43}$ Physics Department, Southern Methodist University, Dallas, Texas, USA

${ }^{44}$ Physics Department, University of Texas at Dallas, Richardson, Texas, USA

${ }^{45}$ DESY, Hamburg and Zeuthen, Hamburg and Berlin, Germany

${ }^{46}$ Lehrstuhl für Experimentelle Physik IV, Technische Universität Dortmund, Dortmund, Germany

${ }^{47}$ Institut für Kern-und Teilchenphysik, Technische Universität Dresden, Dresden, Germany

${ }^{48}$ Department of Physics, Duke University, Durham, North Carolina, USA

${ }^{49}$ SUPA-School of Physics and Astronomy, University of Edinburgh, Edinburgh, United Kingdom

${ }^{50}$ INFN e Laboratori Nazionali di Frascati, Frascati, Italy

${ }^{51}$ Fakultät für Mathematik und Physik, Albert-Ludwigs-Universität, Freiburg, Germany

${ }^{52}$ Departement de Physique Nucleaire et Corpusculaire, Université de Genève, Geneva, Switzerland

${ }^{53 a}$ INFN Sezione di Genova, Genova, Italy

${ }^{53 \mathrm{~b}}$ Dipartimento di Fisica, Università di Genova, Genova, Italy

${ }^{54 a}$ E. Andronikashvili Institute of Physics, Iv. Javakhishvili Tbilisi State University, Tbilisi, Georgia

${ }^{54 \mathrm{~b}}$ High Energy Physics Institute, Tbilisi State University, Tbilisi, Georgia

${ }^{55}$ II Physikalisches Institut, Justus-Liebig-Universität Giessen, Giessen, Germany

${ }^{56}$ SUPA-School of Physics and Astronomy, University of Glasgow, Glasgow, United Kingdom

${ }^{57}$ Laboratoire de Physique Subatomique et de Cosmologie, Université Grenoble-Alpes, CNRS/IN2P3, Grenoble, France

${ }^{58}$ II Physikalisches Institut, Georg-August-Universität, Göttingen, Germany

${ }^{59}$ Laboratory for Particle Physics and Cosmology, Harvard University, Cambridge, Massachusetts, USA

${ }^{60 \mathrm{a}}$ Kirchhoff-Institut für Physik, Ruprecht-Karls-Universität Heidelberg, Heidelberg, Germany

${ }^{60 \mathrm{~b}}$ Physikalisches Institut, Ruprecht-Karls-Universität Heidelberg, Heidelberg, Germany

${ }^{61}$ Faculty of Applied Information Science, Hiroshima Institute of Technology, Hiroshima, Japan

${ }^{62 \mathrm{a}}$ Department of Physics, The Chinese University of Hong Kong, Shatin, N.T., Hong Kong, China

${ }^{62 \mathrm{~b}}$ Department of Physics, The University of Hong Kong, Hong Kong, China

${ }^{62 c}$ Department of Physics and Institute for Advanced Study, The Hong Kong University of Science and Technology, Clear Water Bay, Kowloon, Hong Kong, China

${ }^{63}$ Department of Physics, National Tsing Hua University, Hsinchu, Taiwan

${ }^{64}$ Department of Physics, Indiana University, Bloomington, Indiana, USA

${ }^{65}$ Institut für Astro-und Teilchenphysik, Leopold-Franzens-Universität, Innsbruck, Austria

${ }^{66}$ University of Iowa, Iowa City, Iowa, USA
} 


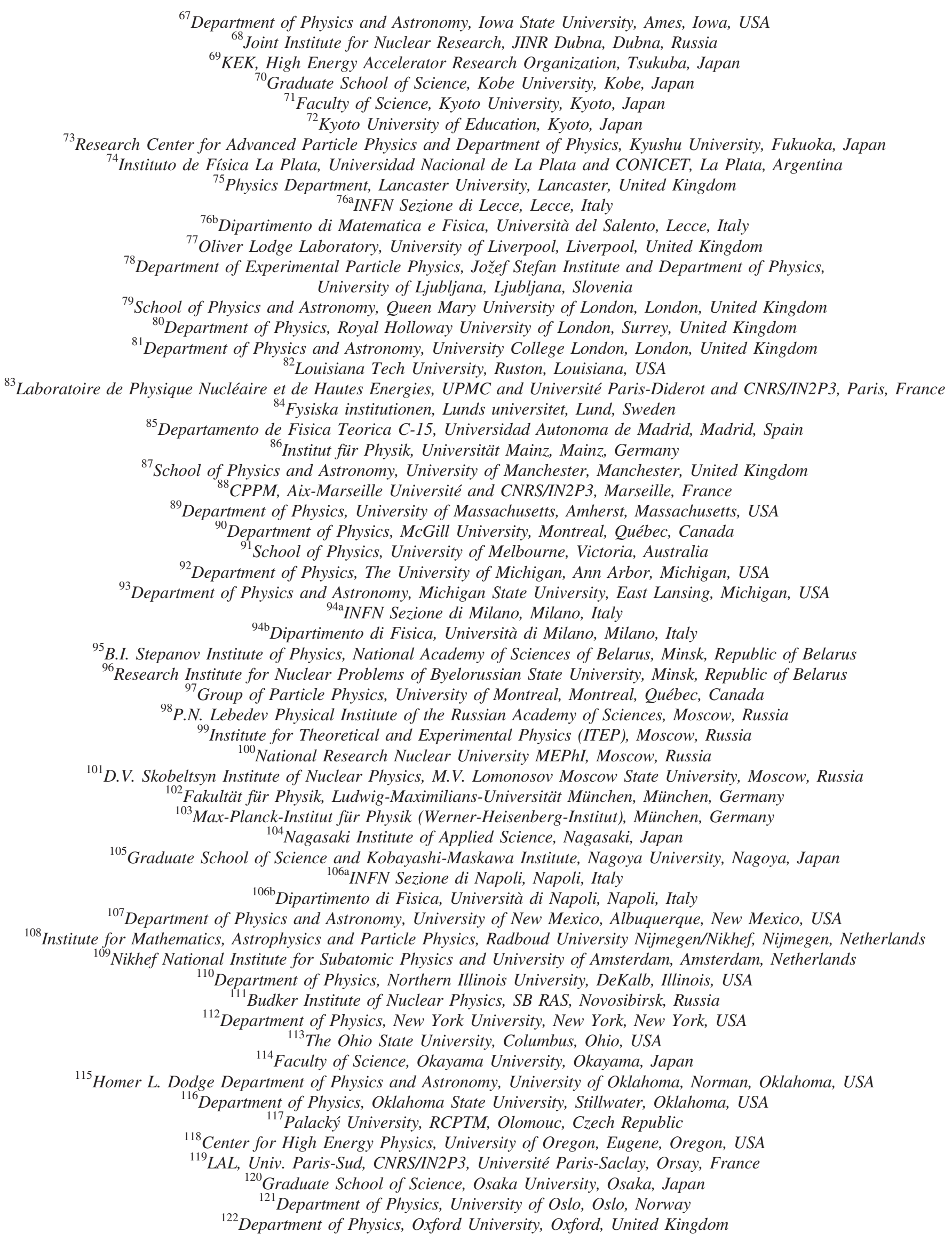




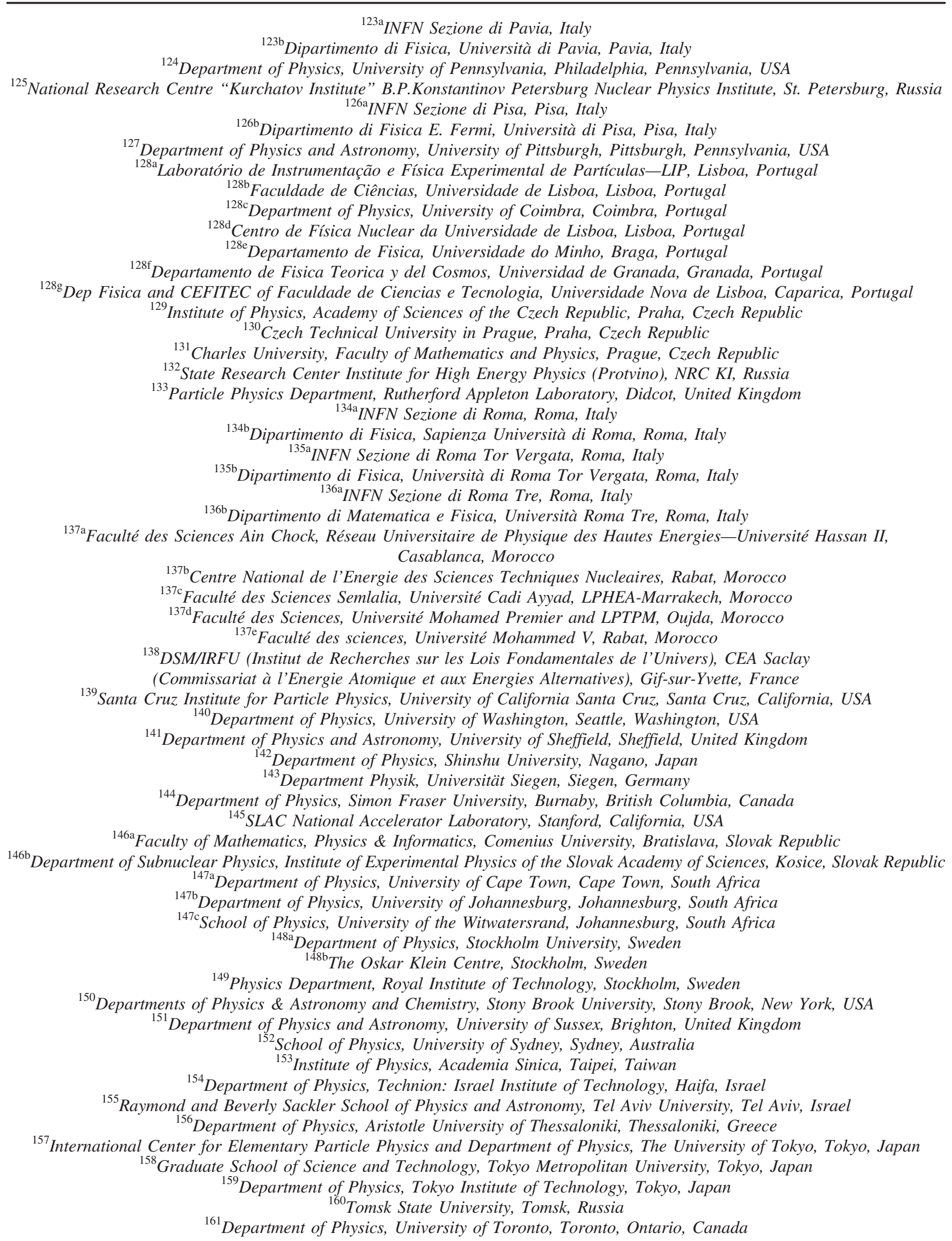




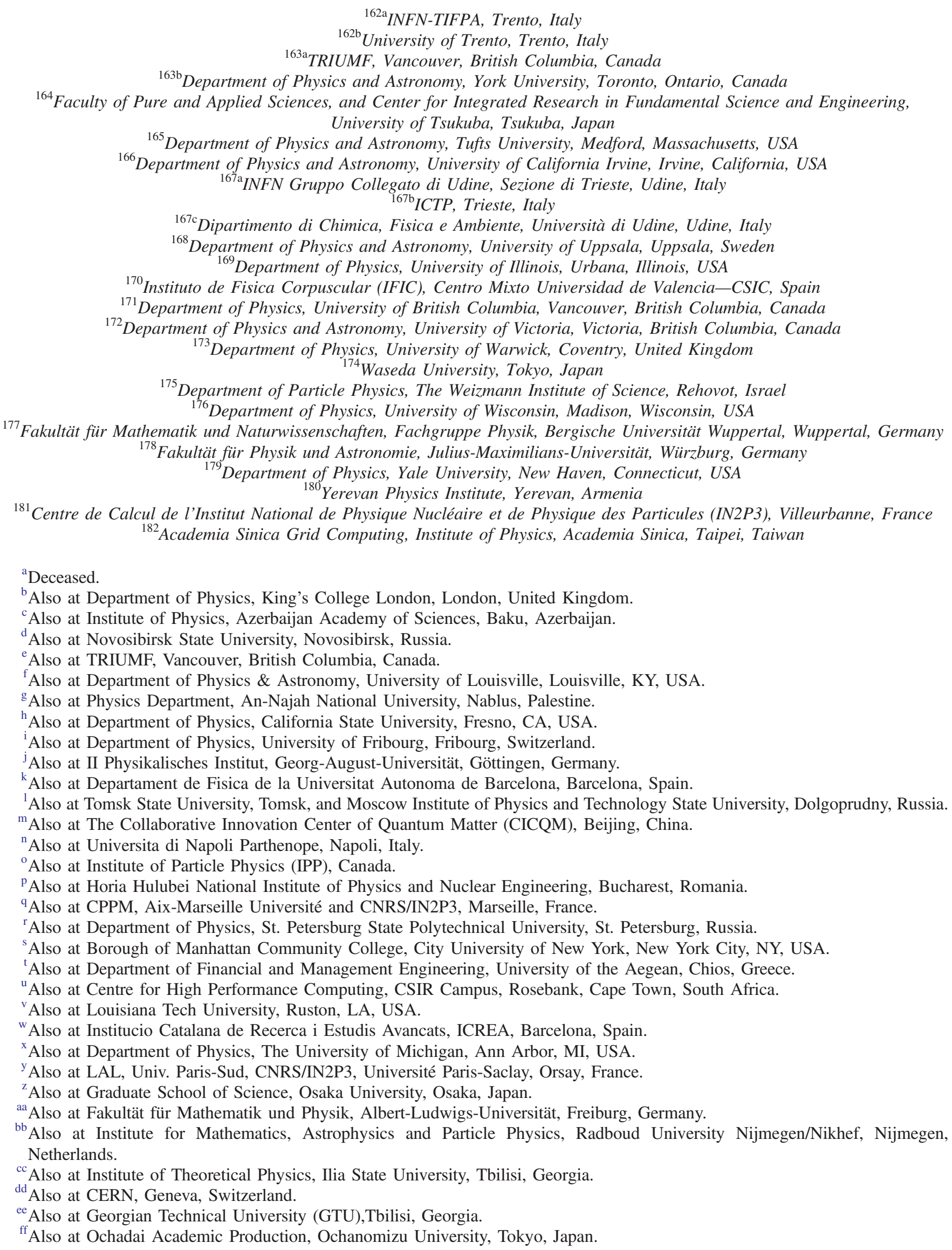


${ }^{\mathrm{gg}}$ Also at Manhattan College, New York, NY, USA.

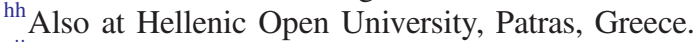

${ }^{\text {ii }}$ Also at The City College of New York, New York, NY, USA.

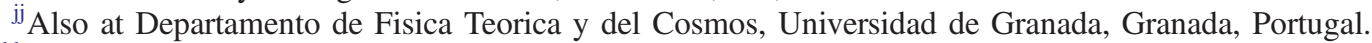

${ }^{\mathrm{kk}}$ Also at Department of Physics, California State University, Sacramento, CA, USA.

${ }^{11}$ Also at Moscow Institute of Physics and Technology State University, Dolgoprudny, Russia.

${ }^{\mathrm{mm}}$ Also at Departement de Physique Nucleaire et Corpusculaire, Université de Genève, Geneva, Switzerland.

${ }^{n n}$ Also at Department of Physics, The University of Texas at Austin, Austin, TX, USA.

${ }^{\circ 0}$ Also at Institut de Física d'Altes Energies (IFAE), The Barcelona Institute of Science and Technology, Barcelona, Spain.

${ }^{\mathrm{pp}}$ Also at School of Physics, Sun Yat-sen University, Guangzhou, China.

${ }^{\mathrm{qq}}$ Also at Institute for Nuclear Research and Nuclear Energy (INRNE) of the Bulgarian Academy of Sciences, Sofia, Bulgaria.

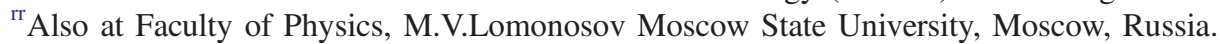

${ }^{\text {ss }}$ Also at National Research Nuclear University MEPhI, Moscow, Russia.

"Also at Department of Physics, Stanford University, Stanford, CA, USA.

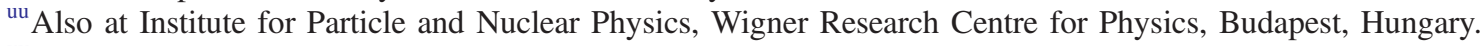

${ }^{\mathrm{v}}$ Also at Giresun University, Faculty of Engineering, Turkey.

${ }^{w w}$ Also at Institute of Physics, Academia Sinica, Taipei, Taiwan.

${ }^{\mathrm{xx}}$ Also at University of Malaya, Department of Physics, Kuala Lumpur, Malaysia.

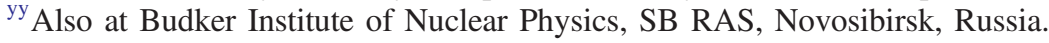

